TRANSACTIONS OF THE

AMERICAN MATHEMATICAL SOCIETY

Volume 351, Number 8, Pages 3119-3141

S 0002-9947(99)02411-3

Article electronically published on March 29, 1999

\title{
THE MAXIMALITY OF THE CORE MODEL
}

\author{
E. SCHIMMERLING AND J. R. STEEL
}

\begin{abstract}
Our main results are: 1) every countably certified extender that coheres with the core model $K$ is on the extender sequence of $K, 2$ ) $K$ computes successors of weakly compact cardinals correctly, 3 ) every model on the maximal 1-small construction is an iterate of $K, 4$ ) (joint with W. J. Mitchell) $K \| \kappa$ is universal for mice of height $\leq \kappa$ whenever $\left.\kappa \geq \aleph_{2}, 5\right)$ if there is a $\kappa$ such that $\kappa$ is either a singular countably closed cardinal or a weakly compact cardinal, and $\square_{\kappa}^{<\omega}$ fails, then there are inner models with Woodin cardinals, and 6) an $\omega$-Erdös cardinal suffices to develop the basic theory of $K$.
\end{abstract}

\section{INTRODUCTION}

This paper deals with the core model, or $K$, as defined in [St1]. Our main results are interesting as part of the pure theory of this model, but they also yield some new consistency strength lower bounds, as we shall point out. Of course, one cannot give a sensible definition of $K$, much less prove anything about it, without an anti-large-cardinal hypothesis. For the most part, the hypothesis of this nature, that we shall assume here, is that there is no inner model satisfying "There is a Woodin cardinal". The results we shall prove about $K$ under this hypothesis have all been proved by others under more restrictive anti-large-cardinal hypotheses, and our proofs build on those earlier proofs.

The term "the core model" was introduced by Dodd and Jensen to mean, roughly, "the largest canonical inner model there is". They gave a precise definition of the term which captures this intuition in the case where there is no inner model with a measurable cardinal in [DoJ]. The work of Mitchell, and then Martin, Mitchell and Steel, led to a precise definition which captures the intuition in the case where there is no inner model with a Woodin cardinal, and every set has a sharp. (Cf. [St1]. The hypothesis that every set has a sharp seems to be a weakness in the basic theory of the core model for a Woodin cardinal. Theorem 5.1 shows how a weaker Erdös partition property suffices.) The canonicity of the core model is evidenced by the absoluteness of its definition: if $G$ is set generic over $V$, then $K^{V}=K^{V[G]}$. (Cf. [St1, 7.3].) The maximality of the core model manifests itself in several ways. Perhaps the most important of these is the weak covering property of $K$, that it computes successors of singular cardinals correctly. (Cf. [St1, 8.15], [MiSchSt], [MiSch], and [SchW].) Other manifestations of the maximality of $K$ are its $\Sigma_{3}^{1}$ correctness (cf. [St1, 7.9]), and its rigidity (cf. [St1, 8.8]).

Received by the editors May 17, 1997 and, in revised form, October 25, 1997.

1991 Mathematics Subject Classification. Primary 03E35, 03E45, 03E55.

Key words and phrases. Large cardinals, core models.

This research was partially supported by the NSF. 
Our main result here is that $K$ is maximal in another sense, namely, that every extender which "could be added" to $K$ is already in $K$. More precisely, Theorem 2.3 says that $K$ is maximal in the sense that any countably certified extender that coheres with $K$ is actually on the extender sequence of $K$, and similarly for the iterates of $K$. Mitchell showed earlier that every countably complete $K$-ultrafilter is in $K$ under the stronger hypothesis that there is no inner model with a measurable cardinal $\kappa$ with $o(\kappa)=\kappa^{++}$. (Cf. [Mi].) It is not known if Theorem 2.3 holds for countably closed extenders. Theorem 2.5 is a result similar to Theorem 2.3 for mice with sufficiently small projectum.

Our first application of Theorem 2.3 is Theorem 3.1, which says that $K$ computes successors of weakly compact cardinals correctly. This extends Kunen's result for $L$ and the weak covering properties of $K$ mentioned above.

Recall from [St1] that $K$ is the transitive collapse of an elementary substructure of $K^{c}$, the background certified core model, and that $K^{c}$ is the limit of the maximal, 1 -small construction $\mathbb{C}$. In Theorem 3.2, another application of Theorem 2.3, we show that $K^{c}$ and all the models on $\mathbb{C}$ are iterates of $K$.

Theorem 3.2 is used to prove Theorem 3.3, which says, roughly, that a Mahlo cardinal is a kind of closure point of the construction $\mathbb{C}$ (although not in as strong a sense as we would like).

The universality of initial segments of $K$ is related to some open questions in descriptive set theory, such as the consistency strength of $u_{2}=\omega_{2}$. Jensen showed that it is consistent for a countable mouse to iterate past $K \| \omega_{1}$, so that $K \| \omega_{1}$ need not be universal for mice of height $\leq \omega_{1}$. Theorem 3.4, another application of maximality, shows that $K \| \kappa$ is universal for mice of height $\leq \kappa$ whenever $\kappa$ is a cardinal $\geq \omega_{2}$. (This result is joint with W. J. Mitchell.)

Using Theorem 3.1 and [Sch2], we prove Theorem 4.1, which says that if $\kappa$ is a weakly compact cardinal and $\square_{\kappa}^{<\omega}$ fails, then there is an inner model with a Woodin cardinal. We recall the definition of $\square_{\kappa}^{<\omega}$ in Section 4; it is weaker than Jensen's principle $\square_{\kappa}$, and stronger than his $\square_{\kappa}^{*}$. By combining our work with his core model induction, Woodin has shown that if there is a weakly compact cardinal $\kappa$ such that $\square_{\kappa}^{<\omega}$ fails, then there is an inner model with $\omega$ Woodin cardinals. We conjecture that the existence of such a $\kappa$ implies that there is an inner model with a superstrong cardinal. Less ambitiously, one might aim to construct an inner model with a cardinal which is strong past a Woodin cardinal, that being the frontier of basic inner model theory at the moment.

The proof of Theorem 4.1, together with the results in [Sch2] and [MiSchSt], are used in the proof of Theorem 4.2, where we show that if $\kappa$ is a singular countably closed cardinal and $\square_{\kappa}^{<\omega}$ fails, then there is an inner model with a Woodin cardinal. With little additional work, the conclusion can be strengthened to the existence of an inner model with $n$ Woodin cardinals for any $n<\omega$. Woodin's core model induction does not seem to yield an inner model with $\omega$ Woodin cardinals in this situation. Again, we conjecture that the existence of such a $\kappa$ implies that there is an inner model with a superstrong cardinal.

Some of the theorems in this paper have the hypothesis that $\Omega$ is a measurable cardinal. This is used to see that the theory of [St1] applies (sharps for bounded subsets of $\Omega$ would do). For our theorems that have this hypothesis on $\Omega$, Theorem 5.1 shows how to reduce it to the partition property: $\Omega \longrightarrow(\omega)_{\alpha}^{<\omega}$ for all $\alpha<\Omega$. For example, if it exists, the Erdös cardinal $\kappa(\omega)$ has this property, and it is consistent with $V=L$ that $\kappa(\omega)$ exists. 


\section{Maximality}

Definition 2.1. Suppose that $\mathcal{M}$ is a premouse and that $F$ is an extender that coheres with $\mathcal{M}$. Let $\kappa=\operatorname{crit}(F)$ and $\nu=\nu(F)$. Suppose that

$$
\mathcal{A} \subseteq \bigcup_{n<\omega} \mathcal{P}\left([\kappa]^{n}\right)^{\mathcal{M}} .
$$

Then a weak $\mathcal{A}$-certificate for $(\mathcal{M}, F)$ is a pair $(N, G)$ such that

(a) $N$ is a transitive, power admissible set, ${ }^{\omega} N \subset N$,

$$
\kappa \cup \mathcal{A} \cup\left\{\mathcal{J}_{\kappa}^{\mathcal{M}}\right\} \subset N,
$$

and $G$ is an extender over $N$,

(b) $F \cap\left([\nu]^{<\omega} \times \mathcal{A}\right)=G \cap\left([\nu]^{<\omega} \times \mathcal{A}\right)$,

(c) $V_{\nu+1} \subset \operatorname{ult}(N, G)$,

(d) if $j: N \longrightarrow \operatorname{ult}(N, G)$ is the ultrapower map, then $\mathcal{M}$ and $j\left(\mathcal{J}_{\kappa}^{\mathcal{M}}\right)$ agree below $\operatorname{lh}(F)$, and

(e) $\langle\mathcal{P}(\alpha) \cap N, \in\rangle \prec\langle\mathcal{P}(\alpha), \in\rangle$ whenever $\alpha<\kappa$.

Weak $\mathcal{A}$-certificates are weaker than the $\mathcal{A}$-certificates of [St1, 1.1] in two ways. First, we weakened the condition that $V_{\kappa} \subseteq N$ to just condition (e). Second, $\operatorname{ult}(N, G)$ is not required to be countably closed.

The following definition should be compared with [St1, 1.2].

Definition 2.2. Suppose that $\mathcal{M}$ is a premouse and $F$ is an extender of length $\alpha$ that coheres with $\mathcal{M}$. Then $(\mathcal{M}, F)$ is weakly countably certified iff for all countable

$$
\mathcal{A} \subseteq \bigcup_{n<\omega} \mathcal{P}\left([\kappa]^{n}\right) \cap J_{\alpha}^{\mathcal{M}}
$$

there is a weak $\mathcal{A}$-certificate for $\left(\mathcal{J}_{\alpha}^{\mathcal{M}}, F\right)$.

When $\mathcal{T}$ is an iteration tree, we shall sometimes write $\mathcal{M}(\mathcal{T}, \eta)$ for $\mathcal{M}_{\eta}^{\mathcal{T}}$, the $\eta$ th model of $\mathcal{T}$, to avoid double superscripts. When $\mathcal{T}$ has a final model, we shall denote it either by $\mathcal{M}_{\infty}^{\mathcal{T}}$ or by $\mathcal{M}(\mathcal{T}, \infty)$. The following is our main maximality theorem.

Theorem 2.3. Suppose that $\Omega$ is a measurable cardinal and that there is no inner model with a Woodin cardinal. Let $\mathcal{T}$ be a normal iteration tree on $K$ of successor length $<\Omega$. Suppose that $F$ is an extender that coheres with $\mathcal{M}_{\infty}^{\mathcal{T}}$ and that

$$
\operatorname{lh}\left(E_{\eta}^{\mathcal{T}}\right)<\operatorname{lh}(F)
$$

for all $\eta<\operatorname{lh}(\mathcal{T})$. Suppose that $\left(\mathcal{M}_{\infty}^{\mathcal{T}}, F\right)$ is weakly countably certified. Then $F$ is on the $\mathcal{M}_{\infty}^{\mathcal{T}}$-sequence.

The following notion will enter into the proof of Theorem 2.3, and is of independent interest.

Definition 2.4. A premouse $\mathcal{M}$ is conditionally $\alpha$-strong iff there is a normal iteration tree $\mathcal{T}$ on an $\alpha$-strong premouse such that $\mathcal{T}$ has successor length, and there is an elementary embedding $\pi$ of $\mathcal{M}$ into an initial segment of $\mathcal{M}_{\infty}^{\mathcal{T}}$ with $\operatorname{crit}(\pi) \geq \alpha$. 
Proof of Theorem 2.3. Fix $\Omega, \mathcal{T}$ and $F$ as in the statement of Theorem 2.3 and let $\kappa=\operatorname{crit}(F), \nu=\nu(F)$, and $\lambda=\ln (F)$.

Let $\mu_{0}$ be an inaccessible cardinal $<\Omega$ such that $F \in V_{\mu_{0}}$. Let $W$ be an $A_{0^{-}}$ soundness witness for $\mathcal{J}_{\mu_{0}}^{K}$. Then $\mathcal{T}$ induces an iteration tree on $W$ with the same extenders, drops, degrees, and tree structure as $\mathcal{T}$, and it is enough to show that $F$ is on the sequence of the final model of the induced tree. For the rest of the proof, we shall write $\mathcal{T}$ for the induced iteration tree on $W$, and never again refer to the original iteration tree on $K$.

As in [St1, 9.7], we write $\Phi(\mathcal{T})$ for the phalanx derived from $\mathcal{T}$. The $F$-extension of $\Phi(\mathcal{T})$ is defined in [St1, 8.5]. By [St1, 8.6], we are done if we show that the $F$-extension of $\Phi(\mathcal{T})$ is $(\Omega+1)$-iterable. (Here we use the hypothesis that $\mathcal{T}$ is normal.) For contradiction, suppose that $\mathcal{U}$ is an ill-behaved iteration tree on the $F$-extension of $\Phi(\mathcal{T})$. We interpret $\mathcal{U}=\emptyset$ to mean that the ultrapower in the definition of the $F$-extension of $\Phi(\mathcal{T})$ is ill-founded. By the tree property at $\Omega$, $\ln (\mathcal{U})<\Omega$.

By the argument of [St1, 6.14], we may fix a successor cardinal $\mu$ such that $\mu_{0}<\mu<\Omega$ and

- there is an iteration tree $\mathcal{S}$ on $\mathcal{J}_{\mu}^{W}$ with the same extenders, drops, degrees, and tree structure as $\mathcal{T}$, such that $\mathcal{M}_{\eta}^{\mathcal{S}}$ is an initial segment of $\mathcal{M}_{\eta}^{\mathcal{T}}$ whenever $\eta<\operatorname{lh}(\mathcal{T})$, and

- there is an ill-behaved iteration tree $\mathcal{V}$ on the $F$-extension of $\Phi(\mathcal{S})$ with the same extenders, drops, degrees, and tree structure as $\mathcal{U}$, such that $\mathcal{M}_{\eta}^{\mathcal{V}}$ is an initial segment of $\mathcal{M}_{\eta}^{\mathcal{U}}$ whenever $\eta<\operatorname{lh}(\mathcal{U})$.

Recall that the $F$-extension of $\Phi(\mathcal{S})$ is the phalanx

$$
\Phi(\mathcal{S}) \frown\left\langle\mathrm{ult}_{k}(\mathcal{P}, F), k, \nu\right\rangle
$$

where $\mathcal{P}$ is the initial segment of $\mathcal{M}_{\gamma}^{\mathcal{S}}$ to which $F$ would be applied according to the rules for $\omega$-maximal iteration trees, and $k<\omega$ is the degree. (This is not literally true, as the official definition of a phalanx, [St1, 9.5], has a fourth coordinate, in our case, either $\nu$ or $\lambda$, depending on the type of $\mathcal{P}$; we shall suppress this fourth coordinate.)

Let $X$ be a countable elementary substructure of $V_{\Omega}$ with $\mathcal{V} \in X$ and let

$$
\pi_{X}: M_{X} \longrightarrow V_{\Omega}
$$

be the corresponding uncollapse. Say $\pi_{X}\left(\mathcal{S}_{X}\right)=\mathcal{S}, \pi_{X}\left(F_{X}\right)=F, \pi_{X}\left(\nu_{X}\right)=\nu$, $\pi_{X}\left(\lambda_{X}\right)=\lambda, \pi_{X}\left(\mathcal{P}_{X}\right)=\mathcal{P}$, and $\pi_{X}\left(\mathcal{V}_{X}\right)=\mathcal{V}$. By the absoluteness argument like that in the proof of [St1, 6.14], $\mathcal{V}_{X}$ is a simple, ill-behaved iteration tree on

$$
\Phi\left(\mathcal{S}_{X}\right) \frown\left\langle\operatorname{ult}_{k}\left(\mathcal{P}_{X}, F_{X}\right), k, \nu_{X}\right\rangle,
$$

the $F_{X}$-extension of $\Phi\left(\mathcal{S}_{X}\right)$.

Let $Y$ be an elementary substructure of $V_{\Omega}$ with

$$
X \cup(\lambda+1) \subset Y
$$

and $\operatorname{card}(Y)=\operatorname{card}(\nu)$. Let $\pi_{Y}: M_{Y} \longrightarrow V_{\Omega}$ be the uncollapse of $Y$ and

$$
\sigma=\pi_{Y}^{-1} \circ \pi_{X}
$$

So $\sigma$ is an elementary embedding of $M_{X}$ into $M_{Y}$ and

$$
\sigma \uparrow(\lambda+1)=\pi_{X} \uparrow(\lambda+1) .
$$


Say $\pi_{Y}\left(\mathcal{S}_{Y}\right)=\mathcal{S}$ and $\pi_{Y}\left(\mathcal{P}_{Y}\right)=\mathcal{P}$. Note that $\mathcal{S}_{Y}$ has the same extenders, drops, degrees, and tree structure as $\mathcal{S}$, and that $\mathcal{M}\left(\mathcal{S}_{Y}, 0\right)$ is crit $\left(\pi_{Y}\right)$-strong.

Let

$$
\mathcal{A}=\operatorname{ran}(\sigma) \cap \bigcup_{n<\omega} \mathcal{P}\left([\kappa]^{n}\right)^{\mathcal{P}_{Y}}
$$

and $(N, G)$ be a weak $\mathcal{A}$-certificate for $\left(\mathcal{P}_{Y}, F\right)$. This is possible because $\left(\mathcal{M}_{\infty}^{\mathcal{T}}, F\right)$ is weakly countably certified, and $\mathcal{P}_{Y}$ and $\mathcal{P}$ agree with $\mathcal{M}_{\infty}^{\mathcal{T}}$ on subsets of $\kappa$. Let $j: N \longrightarrow \operatorname{ult}(N, G)$ be the ultrapower map.

Pick a coordinate $b \in[\nu]^{<\omega}$ together with functions $u \mapsto \nu_{u}, u \mapsto \lambda_{u}$, and $u \mapsto \sigma_{u}$ in $N$ such that

$$
\begin{aligned}
& \nu=\left[b, u \mapsto \nu_{u}\right]_{G}^{N}, \\
& \lambda=\left[b, u \mapsto \lambda_{u}\right]_{G}^{N},
\end{aligned}
$$

and

$$
\sigma=\left[b, u \mapsto \sigma_{u}\right]_{G}^{N} .
$$

This is possible by clause (c) in Definition 2.1. By Łoś' theorem, there is a set $B \in G_{b}$ such that for all $u \in B$,

- $\sigma_{u} \||\mathcal{M}|$ is an elementary embedding of $\mathcal{M}$ into $\sigma_{u}(\mathcal{M})$ for every initial segment $\mathcal{M}$ of a model on $\Phi\left(\mathcal{S}_{X}\right)$,

- $\nu_{u}=\sigma_{u}\left(\nu_{X}\right)<\kappa$, and

- $\lambda_{u}=\sigma_{u}\left(\lambda_{X}\right)<\kappa$.

Lemma 2.4.1. For $G_{b}$-almost every $u \in B$, there is a $\Sigma_{k+1}$-elementary, weak $k$ embedding

$$
\tau: \operatorname{ult}_{k}\left(\mathcal{P}_{X}, F_{X}\right) \longrightarrow \mathcal{P}_{Y}
$$

such that $\tau\left\lceil\lambda_{X}=\sigma_{u}\left\lceil\lambda_{X}\right.\right.$.

Proof. Let $\left\langle f_{n} \mid n<\omega\right\rangle$ be a sequence of $\Sigma_{k}\left(\mathcal{P}_{Y}\right)$-functions and

$$
\left\langle b_{n} \mid n<\omega\right\rangle
$$

be a sequence of coordinates from $[\lambda]^{<\omega}$ such that

$$
\left|\operatorname{ult}_{k}\left(\mathcal{P}_{Y}, F\right)\right| \cap \operatorname{ran}(\sigma)=\left\{\left[b_{n}, f_{n}\right]_{F}^{\mathcal{P}_{Y}} \mid n<\omega\right\} .
$$

We assume that $b=b_{0} \subset b_{n} \subset b_{n+1}$ for all $n<\omega$. We assume that $f_{n}$ is chosen to be a constant function whenever possible. We assume that if

$$
\left[b_{n}, f_{n}\right]_{F}^{\mathcal{P}_{Y}}=\sigma(\xi)
$$

for some $\xi<\lambda_{X}$, then $\sigma(\xi) \in b_{n}$ and $f_{n}$ is the projection function $u \mapsto u^{\sigma(\xi), a}$.

For each $\xi<\lambda_{X}$ and $n \in \omega$ with $\sigma(\xi) \in b_{n}$, let

$$
C_{\xi}^{n}=\left\{u \in[\kappa]^{\left|b_{n}\right|} \mid \sigma_{u^{b_{0}, b_{n}}}(\xi)=u^{\sigma(\xi), b_{n}}\right\} .
$$

Then $C_{\xi}^{n} \in G_{b_{n}}$. Since $N$ is countably closed (or, just because the map $u \mapsto \sigma_{u}$ and all the reals are in $N$ ), it follows that

$$
\left\langle C_{\xi}^{n} \mid \xi<\lambda_{X} \wedge \sigma(\xi) \in b_{n}\right\rangle
$$

is an element of $N$. 
If $n<\omega$ and $e$ is the Gödel number for a $\Sigma_{k}$ formula $\varphi$ such that

$$
\operatorname{ult}_{k}\left(\mathcal{P}_{Y}, F\right) \models \varphi\left[\left[b_{n}, f_{n}\right]_{F}^{\mathcal{P}_{Y}}\right],
$$

then let

$$
T_{e}^{n}=\left\{u \in[\kappa]^{\left|b_{n}\right|} \mid \mathcal{P}_{Y} \models \varphi\left[f_{n}(u)\right]\right\} .
$$

Then, $T_{e}^{n} \in F_{b_{n}} \cap \mathcal{A}$ by Loś' theorem and our choice of $\mathcal{A}$. Moreover, the partial function $(n, e) \mapsto T_{e}^{n}$ is an element of $N$ since $N$ is countably closed.

Let $\left\langle B_{n} \mid n<\omega\right\rangle$ be a sequence in $N$ such that $B_{n} \in G_{b_{n}}$ for all $n<\omega$, with the following three properties. First, $B_{0}=B$. Second, for every $m, e<\omega$ such that $T_{e}^{m}$ is defined, there is an $n \geq m$ with

$$
\left(B_{n}\right)^{b_{m}, b_{n}} \subseteq T_{e}^{m} .
$$

Third, for every $m<\omega$ and $\xi<\lambda_{X}$, there is an $n \geq m$ such that

$$
\left(B_{n}\right)^{b_{m}, b_{n}} \subseteq C_{\xi}^{m} .
$$

We need a "fiber" through the sets $B_{n}$. There is a tree $\mathbb{A}$ in $N$ that searches for a function $g: \omega \longrightarrow[\kappa]^{<\omega}$ such that

$$
g(m) \subset g(n) \in B_{n}
$$

and

$$
\left(b_{n}\right)^{g(m), g(n)}=b_{m}
$$

whenever $m<n<\omega$. Since $\left\langle b_{n} \mid n<\omega\right\rangle$ is a branch through $j(\mathbb{A})_{b}$, there is a branch through $j(\mathbb{A})_{b}$ in $\operatorname{ult}(N, G)$. Hence, for $G_{b}$-almost every $u \in B$, there is a branch $g$ through $\mathbb{A}_{u}$ in $N$.

Given $u \in B$, fix $g \in N$ such that $g$ is a branch through $\mathbb{A}_{u}$, and define a map

$$
\tau: \operatorname{ult}_{k}\left(\mathcal{P}_{X}, F_{X}\right) \longrightarrow \mathcal{P}_{Y}
$$

by

$$
\tau\left(\sigma^{-1}\left(\left[b_{n}, f_{n}\right]_{F}^{\mathcal{P}_{Y}}\right)\right)=f_{n}(g(n)) .
$$

Because we "met" the sets $T_{e}^{n}, \tau$ is a weak $k$-embedding of $\operatorname{ult}_{k}\left(\mathcal{P}_{X}, F_{X}\right)$ into $\mathcal{P}_{Y}$ (recall that weak $k$-embeddings need not be $\Sigma_{k+1}$-elementary). Because $f_{n}$ was chosen to be the constant function whenever possible, if

$$
i_{X}: \mathcal{P}_{X} \longrightarrow \operatorname{ult}_{k}\left(\mathcal{P}_{X}, F_{X}\right)
$$

is the ultrapower map, then $\tau \circ i_{X}=\sigma$. Since $i$ is a $k$-embedding and $\sigma$ is elementary, $\tau$ is a $\Sigma_{k+1}$-elementary. Because we "met" the sets $C_{\xi}^{n}$, it follows that

$$
\tau\left\lceil\lambda_{X}=\sigma_{u}\left\lceil\lambda_{X} .\right.\right.
$$

Lemma 2.4.2. There are functions $u \mapsto \mathcal{P}_{u}$ and $u \mapsto \tau_{u}$ in $N$ such that for $G_{b^{-}}$ almost every $u \in B$, both $V$ and $N$ satisfy

(a) $\mathcal{P}_{u}$ is a conditionally $\left(\lambda_{u}+1\right)$-strong premouse,

(b) $\mathcal{P}_{u}$ and $\mathcal{J}_{\kappa}^{\mathcal{P}}$ agree below $\lambda_{u}+1$, and

(c) $\tau_{u}$ is a $\Sigma_{k+1}$-elementary, weak $k$-embedding of

$$
\mathrm{ult}_{k}\left(\mathcal{P}_{X}, F_{X}\right)
$$

into $\mathcal{P}_{u}$ such that $\tau_{u}\left\lceil\lambda_{X}=\sigma_{u}\left\lceil\lambda_{X}\right.\right.$. 
Proof. First note that $\mathcal{P}_{Y}$ is conditionally $(\lambda+1)$-strong as witnessed by an initial segment of the iteration tree $\mathcal{S}_{Y}$ and the identity map on $\mathcal{P}_{Y}$.

Given $u \in B$ and a corresponding $\tau$ as in Lemma 2.4.1, let

$$
\mathcal{P}_{u}^{*}=\mathcal{H}_{\omega}^{\mathcal{P}_{Y}}\left(\left(\lambda_{u}+1\right) \cup \operatorname{ran}(\tau)\right),
$$

and let

$$
\tau_{u}^{*}: \operatorname{ult}_{k}\left(\mathcal{P}_{X}, F_{X}\right) \longrightarrow \mathcal{P}_{u}^{*}
$$

be the corresponding collapse of $\tau$. Then the functions $u \mapsto \mathcal{P}_{u}^{*}$ and $u \mapsto \tau_{u}^{*}$ satisfy conditions (a), (b), and (c) in $V$, but these functions are not necessarily in $N$.

By the elementarity given in clause (e) of Definition 2.1, for each appropriate $u \in B$, there is a premouse $\mathcal{P}_{u}$ and an embedding $\tau_{u}$, both in $N$, which satisfy conditions (a), (b), and (c) of Lemma 2.4.2 in both $N$ and $V$. Since $N$ satisfies AC, there are appropriate functions $u \mapsto \mathcal{P}_{u}$ and $u \mapsto \tau_{u}$ in $N$.

Fix functions as in Lemma 2.4.2 and let

$$
\mathcal{P}^{*}=\left[b, u \mapsto \mathcal{P}_{u}\right]_{G}^{N}
$$

and

$$
\tau^{*}=\left[b, u \mapsto \tau_{u}\right]_{G}^{N} .
$$

Lemma 2.4.3. $\tau^{*}$ is a $\Sigma_{k+1}$-elementary, weak $k$-embedding of

$$
\operatorname{ult}_{k}\left(\mathcal{P}_{X}, F_{X}\right)
$$

into $\mathcal{P}^{*}$, and $\tau^{*}\left|\lambda_{X}=\sigma\right| \lambda_{X}$.

Proof. Immediate from the definitions of $\mathcal{P}^{*}$ and $\tau$, clause (c) of Lemma 2.4.2, and Łoś' theorem.

Lemma 2.4.4. $\mathcal{M}\left(\mathcal{S}_{Y}, \infty\right)$ and $\mathcal{P}^{*}$ agree below $\lambda$.

Proof. Clearly $\mathcal{M}\left(\mathcal{S}_{Y}, \infty\right), \mathcal{M}(\mathcal{S}, \infty)$, and $\mathcal{M}(\mathcal{T}, \infty)$ all agree beyond $\lambda$. By coherence, $\mathcal{M}\left(\mathcal{S}_{Y}, \infty\right)$ and ult $\left(\mathcal{P}_{Y}, F\right)$ agree below $\lambda$. Clearly $\mathcal{M}\left(\mathcal{S}_{Y}, \infty\right)$ and $\mathcal{P}_{Y}$ agree below $\kappa$. Hence, by clause (d) of Definition 2.1, $\mathcal{M}\left(\mathcal{S}_{Y}, \infty\right)$ and $j\left(\mathcal{J}_{\kappa}^{\mathcal{P}_{Y}}\right)$ agree below $\lambda$. By clause (b) of Lemma 2.4.2, $\mathcal{P}_{u}$ agrees with $\mathcal{J}_{\kappa}^{\mathcal{P}}$ beyond $\lambda_{u}$ for $G_{b}$-almost every $u \in B$. It follows by Loś' theorem that $\mathcal{M}\left(\mathcal{S}_{Y}, \infty\right)$ agrees with $\mathcal{P}^{*}$ below $\lambda$.

Lemma 2.4.5. $\mathcal{P}^{*}$ is conditionally $(\lambda+1)$-strong. Moreover, if $\mathcal{S}^{*}$ is an iteration tree which witnesses that $\mathcal{P}^{*}$ is conditionally $(\lambda+1)$-strong, then $\mathcal{S}^{*}$ uses the same extenders as $\mathcal{S}_{Y}$ below $\lambda$.

Proof. By Łoś' theorem and clause (a) of Lemma 2.4.2, $\mathcal{P}^{*}$ is conditionally $(\lambda+1)$ strong in ult $(N, G)$. By clause (c) of Definition 2.1, $V_{\nu+1} \subset \operatorname{ult}(N, G)$. It follows that $\mathcal{P}^{*}$ is conditionally $(\lambda+1)$-strong in $V$. In light of Lemma 2.4.4, any iteration tree $\mathcal{S}^{*}$ witnessing that $\mathcal{P}^{*}$ is conditionally $(\lambda+1)$-strong must use the same extenders as $\mathcal{S}_{Y}$ below $\lambda$.

Lemma 2.4.6. $\Phi\left(\mathcal{S}_{Y}\right) \frown\left\langle\mathcal{P}^{*}, k, \nu\right\rangle$ is an iterable phalanx.

Proof. Put $\mathfrak{A}=\Phi\left(\mathcal{S}_{Y}\right) \frown\left\langle\mathcal{P}^{*}, k, \nu\right\rangle$. That $\mathfrak{A}$ is a phalanx follows from Lemma 2.4.4.

Fix $\mathcal{S}^{*}$ and an embedding $\psi$ of $\mathcal{P}^{*}$ into an initial segment of $\mathcal{M}\left(\mathcal{S}^{*}, \infty\right)$ which witness that $\mathcal{P}^{*}$ is conditionally $(\lambda+1)$-strong.

Let us write $\psi\left(\mathcal{P}^{*}\right)$ for the initial segment of $\mathcal{M}\left(\mathcal{S}^{*}, \infty\right)$ into which $\psi$ elementarily embeds $\mathcal{P}^{*}$. Since $\mathcal{M}\left(\mathcal{S}^{*}, 0\right)$ is $(\lambda+1)$-strong, there is 
- a successor-length iteration tree $\mathcal{R}$ on $W$, such that $\ln \left(E_{\eta}^{\mathcal{R}}\right)>\lambda$ whenever $\eta<\operatorname{lh}(\mathcal{R})$, and

- an elementary embedding $\varphi$ of $\mathcal{M}\left(\mathcal{S}^{*}, 0\right)$ into an initial segment of $\mathcal{M}_{\infty}^{\mathcal{R}}$ such that $\operatorname{crit}(\varphi)>\lambda$.

We may use $\varphi$ to copy $\mathcal{S}^{*}$ to an iteration tree $\varphi \mathcal{S}^{*}$ on $\mathcal{M}_{\infty}^{\mathcal{R}}$. Let

$$
\varphi_{\infty}: \mathcal{M}\left(\mathcal{S}^{*}, \infty\right) \longrightarrow \mathcal{M}\left(\varphi \mathcal{S}^{*}, \infty\right)
$$

be the final map in the aforementioned copying construction. Then $\operatorname{crit}\left(\varphi_{\infty}\right)>\lambda$ and $\varphi \mathcal{S}^{*}$ uses the same extenders as $\mathcal{S}_{Y}$ below $\lambda$. Therefore $\varphi_{\infty} \circ \psi$ is an elementary embedding of $\mathcal{P}^{*}$ into the initial segment $\left(\varphi_{\infty} \circ \psi\right)\left(\mathcal{P}^{*}\right)$ of $\mathcal{M}\left(\varphi \mathcal{S}^{*}, \infty\right)$ and

$$
\operatorname{crit}\left(\varphi_{\infty} \circ \psi\right)>\lambda \text {. }
$$

Recall that $\mathcal{T}$ was our original iteration tree on $W$. Put

$$
\mathfrak{B}=\Phi(\mathcal{T}) \frown\left\langle\left(\varphi_{\infty} \circ \psi\right)\left(\mathcal{P}^{*}\right), k, \nu\right\rangle
$$

The agreement described above implies that $\mathfrak{B}$ is a phalanx. It is a phalanx "derived" from $\mathcal{T}$ and $\mathcal{R} \frown \varphi \mathcal{S}^{*}$, both iteration trees on $W$. Such $W$-generated phalanxes are iterable by [St1, 6.9].

But now, the pair of maps $\left(\pi_{Y},\left(\varphi_{\infty} \circ \psi\right)\right)$ can be used in the standard way to reduce the iterability of $\mathfrak{A}$ to that of $\mathfrak{B}$.

Recall that $\mathcal{V}_{X}$ is a simple, ill-behaved iteration tree on

$$
\Phi\left(\mathcal{S}_{X}\right) \frown\left\langle\operatorname{ult}_{k}\left(\mathcal{P}_{X}, F_{X}\right), k, \nu_{X}\right\rangle
$$

Using the pair of maps $\left(\sigma, \tau^{*}\right)$ we can copy $\mathcal{V}_{X}$ to an ill-behaved iteration tree on $\Phi\left(\mathcal{S}_{Y}\right) \frown\left\langle\mathcal{P}^{*}, k, \nu\right\rangle$. But now we have a contradiction of Lemma 2.4.6. This completes the proof of Theorem 2.3.

We conclude this section with a version of Theorem 2.3 for mice with sufficiently small projectum. Unlike Theorem 2.3, Theorem 2.5 does not have as a hypothesis that there is no inner model with a Woodin cardinal, nor any such anti-largecardinal hypothesis. For this reason, Theorem 2.5 is applicable to certain core models beyond one Woodin cardinal.

Theorem 2.5. Let $\mathcal{M}$ be a sound premouse such that

$$
\rho_{\omega}(\mathcal{M}) \leq \operatorname{crit}(E)
$$

whenever $E$ is an extender from the $\mathcal{M}$-sequence. Suppose that every countable phalanx which is realizable in an $\mathcal{M}$-based phalanx is $\left(\omega_{1}+1\right)$-iterable. Let $\mathcal{T}$ be a normal iteration tree on $\mathcal{M}$. Suppose that $F$ is an extender that coheres with $\mathcal{M}_{\infty}^{\mathcal{T}}$ such that

$$
\operatorname{lh}\left(E_{\eta}^{\mathcal{T}}\right)<\operatorname{lh}(F)
$$

for all $\eta<\operatorname{lh}(\mathcal{T})$, and such that

$$
\rho_{\omega}(\mathcal{M})<\operatorname{crit}(F)
$$

Suppose that $\left(\mathcal{M}_{\infty}^{\mathcal{T}}, F\right)$ is countably certified. Then $F$ is on the $\mathcal{M}_{\infty}^{\mathcal{T}}$-sequence. 
Sketch. Put $\kappa=\operatorname{crit}(F), \nu=\nu(F)$, and $\lambda=\operatorname{lh}(F)$. Say

$$
\Phi(\mathcal{T}) \frown\langle\operatorname{ult}(\mathcal{P}, F), k, \nu\rangle
$$

is the $F$-extension of $\Phi(\mathcal{T})$. Let $\theta$ be a large regular cardinal and $\pi_{X}: M_{X} \longrightarrow V_{\theta}$ be the uncollapse of a countable elementary substructure $X$ of $V_{\theta}$. Say $\pi_{X}(\overline{\mathcal{T}})=\mathcal{T}$, $\pi_{X}(\bar{F})=F, \pi_{X}(\bar{\nu})=\nu, \pi_{X}(\bar{\lambda})=\lambda$, and $\pi_{X}(\overline{\mathcal{P}})=\mathcal{P}$.

Let $Y$ be a an elementary substructure of $V_{\theta}$ with

$$
X \cup(\lambda+1) \subset Y
$$

and $\operatorname{card}(Y)=\operatorname{card}(\nu)$. Let $\pi_{Y}: M_{Y} \longrightarrow V_{\theta}$ be the uncollapse of $Y$ and let

$$
\sigma=\pi_{Y}^{-1} \circ \pi_{X} .
$$

The hypotheses on soundness and the projectum of $\mathcal{M}$ imply that $\pi_{Y}(\mathcal{T})=\mathcal{T}$, so $\sigma(\overline{\mathcal{T}})=\mathcal{T}$.

Now let

$$
\mathcal{A}=\operatorname{ran}(\sigma) \cap \bigcup_{n<\omega} \mathcal{P}\left([\kappa]^{n}\right)^{\mathcal{P}}
$$

and $(N, G)$ be an $\mathcal{A}$-certificate for $(\mathcal{P}, F)$. (At this point, we could, in fact, take a weak $\mathcal{A}$-certificate for $(\mathcal{P}, F)$ so long as $\mathcal{P} \in N$. Note that $\mathcal{P}$ has cardinality $\leq \kappa$.)

The idea now is to replace " $K$ " by " $\mathcal{M}$ " in proof of Theorem 2.3 to see that the $\bar{F}$-extension of $\Phi(\overline{\mathcal{T}})$ is realizable in an $\mathcal{M}$-based phalanx. More specifically, there is an iteration tree $\mathcal{T}^{*}$ on $\mathcal{M}$ extending $\mathcal{T}$ and a $\Sigma_{k+1}$-elementary, weak $k$-embedding $\tau$ of $\operatorname{ult}_{k}(\overline{\mathcal{P}}, \bar{F})$ into $\mathcal{M}\left(\mathcal{T}^{*}, \infty\right)$ with $\tau\lceil\bar{\lambda}=\sigma\lceil\bar{\lambda}$. (We omit the details.)

By the iterability hypothesis on $\mathcal{M}$, there is a successful coiteration of $\Phi(\overline{\mathcal{T}})$ and the $\bar{F}$-extension of $\Phi(\overline{\mathcal{T}})$. Just as in the proof of [St1, 8.6], our hypotheses on soundness and the projectum of $\mathcal{M}$ allow us to conclude that $\bar{F}$ is on the $\mathcal{M}(\overline{\mathcal{T}}, \infty)$ sequence. By the elementarity of $\pi, F$ is on the $\mathcal{M}_{\infty}^{\mathcal{T}}$-sequence.

\section{Applications of maximality}

Kunen showed that if $\kappa$ is a weakly compact cardinal and

$$
\left(\kappa^{+}\right)^{L}<\kappa^{+},
$$

then $0^{\#}$ exists; see [Jech, p.384]. Our first application of Theorem 2.3 is an extension of Kunen's result to the current generation of core models.

Theorem 3.1. Suppose that $\Omega$ is a measurable cardinal, and that there is no inner model with a Woodin cardinal. Let $\kappa$ be a weakly compact cardinal $<\Omega$. Then $\left(\kappa^{+}\right)^{K}=\kappa^{+}$.

The result analogous to Theorem 3.1 was proved for measurable cardinals $\kappa$ in [St1, 8.15], for singular countably closed cardinals in [MiSchSt], and for all singular cardinals in [MiSch]. From [MiSch], we already know that $\left(\kappa^{+}\right)^{K}$ has cofinality at least $\kappa$ whenever $\kappa$ is a cardinal $\geq \aleph_{2}$.

Lemma 3.1.1 will be used in the proofs of Theorems 3.1, 4.1, and 4.2. We remark that if $\kappa$ is a strong limit cardinal, as in Theorems 3.1 and 4.1, then any $A$ coding $V_{\kappa}$ satisfies the conclusion of Lemma 3.1.1.

Lemma 3.1.1. Assume there is no proper class model with a Woodin cardinal, and let $\kappa$ be a cardinal such that $\alpha<\kappa$ implies $\alpha^{\omega} \leq \kappa$, and every subset of $\kappa$ has a 
sharp. Then there is an $A \subseteq \kappa$ such that whenever $B$ and $C$ are subsets of $\kappa$, and $\mathcal{P}$ is a premouse with $A, \mathcal{P} \in L[B] \cap L[C]$, then

$$
(\mathcal{P} \text { is } \kappa \text {-strong })^{L[B]} \Longleftrightarrow(\mathcal{P} \text { is } \kappa \text {-strong })^{L[C]} \text {. }
$$

Moreover, if there is a measurable cardinal greater than $\kappa$, then $K$ and $K^{B}$ agree below $\left(\kappa^{+}\right)^{K^{L[B]}}$ for all $B$ such that $A \in L[B]$.

Proof. Let $\Omega=\kappa^{+}$. Then, for any $A \subseteq \Omega, \Omega$ is an $A$-indiscernible and the theory of $K$ up to $\Omega$ of [St1] applies in $L[A]$. (Cf. [St1, p.58] and $\S 5$ of this paper.)

Inductively we build $A_{\alpha}$, for $\alpha<\kappa$ which are cardinals of a "stable $K$ " we determine along the way; the final $A$ is just the amalgamation of the $A_{\alpha}$ 's.

Suppose we have $A_{\alpha}$ such that for all $B, C \subseteq \kappa$ with $A_{\alpha} \in L[B] \cap L[C], L[B]$ and $L[C]$ agree on which mice in $L[B] \cap L[C]$ are $<\alpha$-strong, and on what $K$ up to $\alpha$ is, and on the fact that $\alpha$ is a cardinal of $K$. Call a premouse $\mathcal{P}$ "stably $<\alpha$-strong" if $\mathcal{P}$ is $<\alpha$-strong in $L[B]$, for some $B$ with $A_{\alpha} \in L[B]$. For each countable $X \subseteq \alpha$ and countable premouse $\mathcal{Q}$, if there is a stably $<\alpha$-strong premouse $\mathcal{P}$ and an embedding $\pi: \mathcal{Q} \longrightarrow \mathcal{P}$ with $X=\operatorname{ran}(\pi) \cap \alpha$, then pick such a $\mathcal{P}$ of size $\leq \kappa$ and set $\mathcal{P}(\alpha, X, \mathcal{Q})=\mathcal{P}$. (It is possible to pick $\mathcal{P}$ of the same size as $\alpha$.) For the $\alpha$ currently under consideration, since $\alpha^{\omega} \leq \kappa$, there are $\leq \kappa$ many such $\mathcal{P}(\alpha, X, \mathcal{Q})$. We take $D \subseteq \kappa$ to code the function $(X, \mathcal{Q}) \mapsto \mathcal{P}(\alpha, X, \mathcal{Q})$ together with $A_{\alpha}$. It is easy to see that if $D \in L[B] \cap L[C]$, then $L[B]$ and $L[C]$ agree on which mice in $L[B] \cap L[C]$ are $\alpha$-strong. [If $\mathcal{R} \in L[B] \cap L[C]$ and $\mathcal{R}$ is not $\alpha$-strong in $L[B]$, then there is a stably $<\alpha$-strong $\mathcal{P}$, an ill-behaved countable iteration tree $\mathcal{U}$ on a countable phalanx $((\mathcal{Q}, \mathcal{S}), \beta)$, and an elementary embedding $\pi$ such that

$$
\pi(((\mathcal{Q}, \mathcal{S}), \beta))=((\mathcal{P}, \mathcal{R}), \alpha)
$$

Let $X=\operatorname{ran}(\pi) \cap \alpha$. By absoluteness, there is an elementary embedding $\tau: \mathcal{S} \longrightarrow \mathcal{R}$ in $L[C]$ such that $\operatorname{ran}(\tau) \cap \alpha=X$. Another application of absoluteness gives an elementary embedding $\sigma: \mathcal{Q} \longrightarrow \mathcal{P}(\alpha, X, \mathcal{Q})$ in $L[C]$ such that $\operatorname{ran}(\sigma) \cap \alpha=X$. But then $\mathcal{R}$ is not $\alpha$-strong in $L[C]$ as witnessed by $(\sigma, \tau) \mathcal{U}$.] Thus we have determined the "stably $\alpha$-strong" mice, and "stable $K$ up to the $\alpha^{+}$of stable $K$ ". Let $\gamma=\alpha^{+}$ of stable $K$, and $A_{\gamma}$ code $D$ together with stable $K$ up to $\gamma$.

Now let $A$ code the join of the $A_{\alpha}$ 's. It is clear from the construction that the first conclusion of Lemma 3.1.1 holds with " $<\kappa$-strong" in place of " $\kappa$-strong".

Suppose that $B, C \subseteq \kappa, \mathcal{R}$ is a premouse, and $A, \mathcal{R} \in L[B] \cap L[C]$. Say $\mathcal{R}$ is not $\kappa$-strong in $L[B]$. We shall show that $\mathcal{R}$ is not $\kappa$-strong in $L[C]$, from which the rest of Lemma 3.1.1 follows easily.

Since $\mathcal{R}$ is not $\kappa$-strong in $L[B]$, there is a stably $<\kappa$-strong premouse $\mathcal{P}$ and and a countable ill-behaved iteration tree $\mathcal{T}$ on the phalanx $((\mathcal{P}, \mathcal{R}), \kappa)$. Let

$$
\vec{\alpha}=\left\langle\alpha_{i} \mid i<\omega\right\rangle
$$

be an increasing sequence of cardinals of the stable $K$ up to $\kappa$ such that for every $\eta<\operatorname{lh}(\mathcal{T})$, if $\operatorname{crit}\left(E_{\eta}^{\mathcal{T}}\right)<\kappa$, then there is an $i<\omega \operatorname{such}$ that $\operatorname{crit}\left(E_{\eta}^{\mathcal{T}}\right)<\alpha_{i}$. (We are assuming that $\kappa$ is a limit cardinal in stable $K$, the other case being simpler.) By taking elementary substructures in $L[B]$, we find a sequence

$$
\overrightarrow{\mathcal{P}}=\left\langle\mathcal{P}_{i} \mid i<\omega\right\rangle
$$

such that, for each $i<\alpha, \mathcal{P}_{i}$ embeds into $\mathcal{P}$ with critical point $>\alpha_{i}$, so $\mathcal{P}_{i}$ is stably $\alpha_{i}$-strong. Let us construe $\mathcal{T}$ as an iteration tree on the phalanx $((\overrightarrow{\mathcal{P}}, \mathcal{R}), \vec{\alpha})$. Thus 
construed, $\mathcal{T}$ is still ill-behaved. Let $M$ be the transitive collapse of a countable elementary substructure of $V_{\Omega}$ and let $\pi: M \longrightarrow V_{\Omega}$ be the uncollapse. Assume that

$$
\pi((\overrightarrow{\mathcal{Q}}, \mathcal{S}), \vec{\beta}))=((\overrightarrow{\mathcal{P}}, \mathcal{R}), \vec{\alpha})
$$

and $\pi(\mathcal{U})=\mathcal{T}$. By elementarity and absoluteness, $\mathcal{U}$ is an ill-behaved iteration tree on $((\overrightarrow{\mathcal{Q}}, \mathcal{S}), \vec{\beta})$. Put $X_{i}=\operatorname{ran}(\pi) \cap \alpha_{i}$.

In $L[C]$, we have the premouse $\mathcal{R}$, the countable ill-behaved iteration tree $\mathcal{U}$ on the countable phalanx $((\overrightarrow{\mathcal{Q}}, \mathcal{S}), \vec{\beta})$, and each of the countable sets $X_{i}$ 's, but not necessarily $\vec{\alpha}$ nor $\vec{X}=\left\langle X_{i} \mid i<\omega\right\rangle$. But, in $L[C]$, there is a tree that simultaneously searches for

- an increasing sequence $\vec{\gamma}=\left\langle\gamma_{i} \mid i<\omega\right\rangle$ of cardinals of stable $K$ up to $\kappa$,

- a sequence $\left\langle Y_{i} \mid i<\omega\right\rangle$ such that, for every $i<\omega, \mathcal{P}\left(\gamma_{i}, Y_{i}, \mathcal{Q}\right)$ is defined, and

- an elementary embedding $\tau: \mathcal{S} \longrightarrow \mathcal{R}$ such that $\operatorname{ran}(\tau) \cap \gamma_{i}=Y_{i}$ and

$$
\tau\left(\beta_{i}\right)=\gamma_{i}
$$

for $i<\omega$.

There is an infinite branch through this tree which is determined by $\vec{\alpha}, \vec{X}$, and $\pi$. By absoluteness, there is a branch through this tree in $L[C]$. So let $\vec{\gamma}_{i}, \vec{Y}$, and $\tau$ be determined by such a branch. By absoluteness, in $L[C]$, there is a sequence $\vec{\sigma}=\left\langle\sigma_{i} \mid i<\omega\right\rangle$ such that $\sigma_{i}$ is an elementary embedding of $\mathcal{Q}$ into $\mathcal{P}\left(\gamma_{i}, Y_{i}, \mathcal{Q}\right)$ for each $i<\omega$.

Let $(\vec{\sigma}, \tau) \mathcal{U}$ denote the iteration tree obtained by copying $\mathcal{U}$ using $(\vec{\sigma}, \tau)$. Now

$$
\left(\left(\left\langle P\left(\gamma_{i}, Y_{i}, \mathcal{Q}_{i}\right) \mid i<\omega\right\rangle, \mathcal{R}\right), \vec{\gamma}\right)
$$

is not iterable in $L[C]$ as witnessed by the ill-behaved iteration tree $(\vec{\sigma}, \tau) \mathcal{U}$. But, for all $i<\omega, P\left(\gamma_{i}, Y_{i}, \mathcal{Q}_{i}\right)$ is stably $<\gamma_{i}$-strong. Therefore $\mathcal{R}$ is not $\kappa$-strong in $L[C]$.

Proof of Theorem 3.1. Let $\kappa$ be a weakly compact cardinal $<\Omega$ and put

$$
\lambda=\left(\kappa^{+}\right)^{K} .
$$

Assume, for contradiction, that $\lambda<\kappa^{+}$.

Let $N$ be a transitive, power admissible set such that ${ }^{\omega} N \subset N$,

$$
V_{\kappa} \cup \mathcal{J}_{\lambda+1}^{K} \subset N,
$$

and $\operatorname{card}(N)=\kappa$. Pick $A \subseteq \kappa$ so that $N \in L[A]$. We assume that $A$ satisfies the conclusion of Lemma 3.1.1. (As remarked earlier, this follows.)

We shall write $K^{A}$ for $K^{L[A]}$. Let $\lambda=\left(\kappa^{+}\right)^{K^{A}}$. By Lemma 3.1.1, $K^{A}$ and $K$ agree below $\lambda$.

Since $A^{\#}$ exists, $\left(\kappa^{+}\right)^{L[A]}<\kappa^{+}$, so card $(\mathcal{P}(\kappa) \cap L[A])=\kappa$. Because $\kappa$ is weakly compact, there is a nonprincipal, $\kappa$-complete $L[A]$-ultrafilter $U$ over $\kappa$; see [Jech, p.384]. Then $\operatorname{ult}(L[A], U)$ is well-founded. Let

$$
j: L[A] \longrightarrow \operatorname{ult}(L[A], U)=L[j(A)]
$$

be the ultrapower map. Then $\operatorname{crit}(j)=\kappa$ and

$$
A=j(A) \cap \kappa \in L[j(A)],
$$


so $L[A] \subseteq L[j(A)]$. We remark that $\mathcal{P}(\kappa) \cap L[j(A)] \not \subset L[A]$ is possible; see [Jech, p.394]. It follows from Lemma 3.1.1 that $\lambda=\left(\kappa^{+}\right)^{K^{j(A)}}$, and that $K^{j(A)}$ and $K$ agree past $\lambda$. Thus $\mathcal{P}(\kappa) \cap K^{j(A)}=\mathcal{P}(\kappa) \cap K^{A}$.

Let $E_{j}$ be the superstrong extender derived from $j$. So, for every $a \in[j(\kappa)]^{<\omega}$,

$$
\left(E_{j}\right)_{a}=\left\{x \subseteq[\kappa]^{|a|} \mid x \in L[A] \wedge a \in j(x)\right\} .
$$

We claim that for every sequence $\left\langle x_{\alpha} \mid \alpha<\kappa\right\rangle$ in $L[A]$,

$$
E_{j} \cap\left([j(\kappa)]^{<\omega} \times\left\{x_{\alpha} \mid \alpha<\kappa\right\}\right) \in L[j(A)] .
$$

The argument is due to Kunen, as in [MiSt, 1.1]: just note that for any $a \in[j(\kappa)]^{<\omega}$,

$$
E_{a}=\left\{x_{\alpha} \subseteq[\kappa]^{|a|} \mid \alpha<\kappa \wedge a \in j\left(x_{\alpha}\right)\right\}
$$

and

$$
\left\langle j\left(x_{\alpha}\right) \mid \alpha<\kappa\right\rangle \in L[j(A)] .
$$

Set

$$
F=E_{j} \cap\left([j(\kappa)]^{<\omega} \times K^{A}\right)
$$

and

$$
G=E_{j} \cap\left([j(\kappa)]^{<\omega} \times N\right) .
$$

Then $\left(K^{j(A)}, F\right)$ and $(N, G)$ are elements of $L[j(A)]$. Moreover, $L[j(A)]$ satisfies the sentence, " $F\lceil\xi$ coheres with $K$ and $(N, G)$ is an $\mathcal{A}$-certificate for $(K, F\lceil\xi)$ where

$$
\mathcal{A}=\bigcup_{n<\omega} \mathcal{P}\left([\kappa]^{n}\right)^{K}
$$

and unbounded many $\xi<j(\kappa)$ ". (For clause (c) of Definition 2.1, simply note that $L[A] \models " V_{\kappa} \subset N "$, so

$$
L[j(A)] \models " V_{j(\kappa)} \subset j(N) ",
$$

and $j(N)$ and $\operatorname{ult}(N, G)$ have the same sets of rank $<j(\kappa)$.) That $\Omega$ is an $L[j(\kappa)]$ indiscernible is enough to see that the conclusion of Theorem 2.3 holds in $L[j(\kappa)]$. Therefore every initial segment of $F$ is on the $K^{j(A)}$ sequence. But then $\kappa$ is a Shelah cardinal in $K^{j(A)}$, contradicting that there is no inner model with a Woodin cardinal.

By definition, $K$ is the transitive collapse of an elementary substructure of $K^{c}$; see [St1, §5]. Our next application of Theorem 2.3 shows that there is an iteration tree on $K$ with last model $K^{c}$. For the statement of Theorem 3.2, recall that $K^{c}$ is defined as the limit of the maximal 1-small construction $\left\langle\mathcal{N}_{\xi} \mid \xi<\Omega\right\rangle$; see [St1, §1].

Theorem 3.2. Assume that $\Omega$ is a measurable cardinal and that there is no inner model with a Woodin cardinal. Let $\alpha \leq \Omega$ and $k<\omega$. Suppose that $(\mathcal{U}, \mathcal{V})$ is the coiteration of

$$
\left(K, \mathfrak{C}_{k}\left(\mathcal{N}_{\xi}\right)\right) .
$$

Then $\mathcal{V}$ is trivial. In particular, since $K^{c}$ is universal, $K^{c}$ is an iterate of $K$. 
Proof. The proof is by induction. Fix $\xi \leq \Omega$ and $k<\omega$. Assume that $\mathfrak{C}_{\ell}\left(\mathcal{N}_{\zeta}\right)$ does not move in its coiteration with $K$ whenever

$$
(\zeta, \ell)<_{l e x}(\xi, k) \text {. }
$$

For contradiction, assume that $\mathfrak{C}_{k}\left(\mathcal{N}_{\xi}\right)$ moves in its coiteration with $K$.

Let $(\mathcal{U}, \mathcal{V})$ be the coiteration of

$$
\left(K, \mathfrak{C}_{k}\left(\mathcal{N}_{\xi}\right)\right)
$$

and $\theta<\operatorname{lh}(\mathcal{V})$ be least such that $E_{\theta}^{\mathcal{V}} \neq \emptyset$. Put $\mathcal{T}=\mathcal{U} \uparrow(\theta+1)$ and $F=E_{\theta}^{\mathcal{V}}$. Then $F$ coheres with $\mathcal{M}_{\theta}^{\mathcal{T}}$ but $F$ is not on the $\mathcal{M}_{\theta}^{\mathcal{T}}$-sequence.

First suppose that $k=0$ and that $F$ is the last extender of $\mathcal{N}_{\xi}$. Then $F$ is countably certified and $\operatorname{crit}(F)$ is an inaccessible cardinal, by the definition of $\mathcal{N}_{\xi}$. Hence, by Theorem 3.2, $F$ is on the $\mathcal{M}_{\theta}^{\mathcal{T}}$-sequence, a contradiction.

Next, suppose that $k=0$ but that $F$ is not the last extender of $\mathcal{N}_{\xi}$. Then there is some $\zeta<\xi$ and $\ell<\omega$ such that $F$ is on the $\mathfrak{C}_{\ell}\left(\mathcal{N}_{\zeta}\right)$-sequence. From the fact that $\mathfrak{C}_{\ell}\left(\mathcal{N}_{\zeta}\right)$ and $\mathfrak{C}_{k}\left(\mathcal{N}_{\xi}\right)$ agree below $\operatorname{lh}(F)+1$, it follows that $\mathfrak{C}_{\ell}\left(\mathcal{N}_{\zeta}\right)$ moves in its coiteration with $K$. This contradicts our induction hypothesis.

So $k>0$. By the induction hypothesis,

$$
\mathfrak{C}_{k-1}\left(\mathcal{N}_{\xi}\right) \neq \mathfrak{C}_{k}\left(\mathcal{N}_{\xi}\right) \text {. }
$$

Thus, $\mathfrak{C}_{k-1}\left(\mathcal{N}_{\xi}\right)$ is $(k-1)$-sound but not $k$-sound. By the induction hypothesis, there is an iteration tree $\mathcal{S}$ on $K$ such that the last model of $\mathcal{S}$ is $\mathfrak{C}_{k-1}\left(\mathcal{N}_{\xi}\right)$. But then $\mathfrak{C}_{k}\left(\mathcal{N}_{\xi}\right)$ is a model along the main branch of $\mathcal{S}$. Namely,

$$
\mathfrak{C}_{k}\left(\mathcal{N}_{\xi}\right)=\left(\mathcal{M}_{\gamma+1}^{*}\right)^{\mathcal{S}}
$$

where $\gamma+1$ is the last drop to an ultrapower of degree at most $k-1$ along the main branch of $\mathcal{S}$. But then $\mathcal{S} \uparrow(\gamma+1)$ witnesses that the theorem holds for $(\xi, k)$, a contradiction.

Recall that $\mathcal{N}_{\kappa}$ is a premouse of height $\kappa$ whenever $\kappa$ is a cardinal, but that $\mathcal{N}_{\kappa}$ need not equal $\mathcal{J}_{\kappa}^{K^{c}}$. Theorem 3.3 appears to be the first step in showing that $\mathcal{N}_{\kappa}=\mathcal{J}_{\kappa}^{K^{c}}$ when $\kappa$ is a Mahlo cardinal, but we do not know if this is true.

Theorem 3.3. Suppose that there is no inner model with a Woodin cardinal and that $\Omega$ is a measurable cardinal. Let $\kappa<\Omega$ be a Mahlo cardinal. Then there is an iteration tree $\mathcal{T}$ on $\mathcal{J}_{\kappa}^{K}$ such that $\mathcal{M}_{\infty}^{\mathcal{T}}=\mathcal{N}_{\kappa}$. In particular,

$$
\left(\alpha^{+}\right)^{\mathcal{N}_{\kappa}}=\alpha^{+}
$$

for all singular cardinals $\alpha<\kappa$, so $\mathcal{N}_{\kappa}$ is universal for mice of height $\leq \kappa$.

Proof. By Theorem 3.2, there is an iteration tree $\mathcal{T}$ on $K$ with $\mathcal{M}_{\infty}^{\mathcal{T}}=\mathcal{N}_{\kappa}$. Certainly $\operatorname{lh}(\mathcal{T}) \leq \kappa+1$. Let us assume that $\operatorname{lh}(\mathcal{T})=\kappa+1$, the other case being clear. It is enough if we show that $\mathcal{T}$ does not drop and that $i_{0, \kappa}^{\mathcal{T}}(\alpha)<\kappa$ for all $\alpha<\kappa$.

Suppose otherwise. Form a continuous chain

$$
\left\langle X_{\alpha} \mid \alpha<\kappa\right\rangle
$$

of elementary submodels of $\left\langle V_{\kappa+1}, \in, \mathcal{T}\right\rangle$ such that $\operatorname{card}\left(X_{\alpha}\right)=\alpha$ and

$$
{ }^{\omega} X_{\alpha} \in X_{\alpha+1}
$$

for all $\alpha<\kappa$. Let

$$
\pi_{\alpha}: P_{\alpha} \longrightarrow V_{\kappa+1}
$$


be the uncollapse of $X_{\alpha}$ and $G_{\alpha}$ be the length $\kappa$ extender derived from $\pi_{\alpha}$ whenever $\alpha<\kappa$. There is a stationary set of inaccessible cardinals $\alpha<\kappa$ such that if $(\beta+1) T \kappa$ and $\alpha=\operatorname{pred}^{T}(\beta+1)$, then $\alpha=\operatorname{crit}\left(\pi_{\alpha}\right), \pi_{\alpha}(\alpha)=\kappa, X_{\alpha}$ is countably closed, $\mathcal{M}_{\alpha}^{\mathcal{T}} \cap \mathcal{P}(\alpha) \in P_{\alpha}$, and $\left(P_{\alpha}, G_{\alpha}\right)$ is an $\mathcal{A}$-certificate for

$$
\left(\mathcal{J}_{l h\left(E_{\beta}^{\mathcal{T}}\right)}^{\mathcal{M}_{\alpha}^{\mathcal{T}}}, E_{\beta}^{\mathcal{T}}\right)
$$

where

$$
\mathcal{A}=\bigcup_{n<\omega} \mathcal{P}\left([\alpha]^{n}\right) \cap\left|\mathcal{M}_{\alpha}^{\mathcal{T}}\right| .
$$

The proof is similar to the standard argument that coiterations of mice terminate. Fix any such $\alpha<\kappa$. Let $\beta$ be such that $\alpha=\operatorname{pred}^{T}(\beta+1)$ and $(\beta+1) T \kappa$. Clearly $E_{\beta}^{\mathcal{T}}$ coheres with $\mathcal{N}_{\kappa}$ and $\left(\mathcal{N}_{\kappa}, E_{\beta}^{\mathcal{T}}\right)$ is countably certified. Note that $\operatorname{lh}\left(E_{\beta}^{\mathcal{T}}\right)$ is a cardinal in $\mathcal{N}_{\kappa}$. Let $\gamma<\kappa$ be the supremum of the $\eta<\kappa$ such that $\rho_{\omega}\left(\mathcal{N}_{\eta}\right)<\operatorname{lh}\left(E_{\beta}^{\mathcal{T}}\right)$. It is not hard to see that $\mathcal{N}_{\gamma}$ is the passive initial segment of $\mathcal{N}_{\kappa}$ of height $\operatorname{lh}\left(E_{\beta}^{\mathcal{T}}\right)$, $\mathcal{N}_{\gamma} \frown\left\langle E_{\beta}^{\mathcal{T}}\right\rangle$ is a premouse, and that $\left(\mathcal{N}_{\gamma}, E_{\beta}^{\mathcal{T}}\right)$ is certified as above. Therefore

$$
\rho_{\omega}\left(\mathcal{N}_{\gamma+1}\right) \leq \nu\left(E_{\beta}^{\mathcal{T}}\right)<\operatorname{lh}\left(E_{\beta}^{\mathcal{T}}\right)
$$

a contradiction.

Theorem 3.4 (with W. J. Mitchell). Suppose that there is no inner model with a Woodin cardinal and that $\Omega$ is a measurable cardinal. Let $\kappa$ be a cardinal such that $\aleph_{2} \leq \kappa<\Omega$. Then $\mathcal{J}_{\kappa}^{K}$ is universal for premice of height $\kappa$.

Theorem 3.4 reduces quickly to the case in which $\kappa$ is a successor cardinal. The authors proved Theorem 3.4 under the assumption that $\kappa$ is a countably closed, successor cardinal. Then, Mitchell saw how to eliminate the countable closure, by adapting the methods of [MiSch].

Sketch of Theorem 3.4. Let $\kappa$ be the least cardinal between $\aleph_{2}$ and $\Omega$ for which Theorem 3.4 fails. Then $\kappa$ is a successor cardinal, say $\kappa=\mu^{+}$.

Suppose that $\mathcal{M}$ is a counterexample to the theorem and that $(\mathcal{S}, \mathcal{T})$ is the coiteration of $\left(\mathcal{J}_{\kappa}^{K}, \mathcal{M}\right)$. Then, there is club set $C \subseteq[0, \kappa]_{T}$ such that

$$
\begin{gathered}
\mathcal{D}^{\mathcal{T}} \cap[\alpha, \kappa]_{T}=\emptyset, \\
\operatorname{crit}\left(i_{\alpha, \kappa}^{\mathcal{T}}\right)=\alpha,
\end{gathered}
$$

and

$$
i_{\alpha, \kappa}^{\mathcal{T}}(\alpha)=\kappa
$$

whenever $\alpha \in C$. Let us also assume that $C \subseteq[0, \kappa]_{S}$ and that

$$
i_{0, \alpha}^{\mathcal{S}}{ }^{\prime \prime} \alpha \subseteq \alpha \text {. }
$$

Define $\beta(\alpha)$ to be the $T$-successor of $\alpha$ in $[0, \kappa]_{T}$.

First assume that $\mu^{\omega}=\mu$. Form a continuous chain

$$
\left\langle X_{\alpha} \mid \alpha<\kappa\right\rangle
$$

of elementary submodels of $\left\langle V_{\Omega+\omega}, \in, \mathcal{T}\right\rangle$ such that $\operatorname{card}\left(X_{\alpha}\right)=\mu$,

$$
{ }^{\omega} X_{\alpha} \in X_{\alpha+1}
$$


and $X_{\alpha} \cap \kappa \in \kappa$ for all $\alpha<\kappa$. Let

$$
\pi_{\alpha}: P_{\alpha} \longrightarrow V_{\Omega+\omega}
$$

be the uncollapse of $X_{\alpha}$ and let $G_{\alpha}$ be the length $\kappa$ extender derived from $\pi_{\alpha}$. Then, as in the proof of Theorem 3.3, for a stationary set of $\alpha \in C,\left(P_{\alpha}, G_{\alpha}\right)$ is a weak $\mathcal{A}$-certificate for

$$
\left(\mathcal{J}_{l h\left(E_{\beta}^{\mathcal{T}}\right)}^{\mathcal{M}_{\mathcal{T}}^{\mathcal{T}}}, E_{\beta}^{\mathcal{T}}\right)
$$

where $\beta=\beta(\alpha)$ and

$$
\mathcal{A}=\bigcup_{n<\omega} \mathcal{P}\left([\alpha]^{n}\right) \cap\left|\mathcal{M}_{\alpha}^{\mathcal{T}}\right| .
$$

It follows that $\left(\mathcal{M}_{\beta}^{\mathcal{S}}, E_{\beta}^{\mathcal{T}}\right)$ is weakly countably certified. (Note that $\left(P_{\alpha}, G_{\alpha}\right)$ cannot be an $\mathcal{A}$-certificate since $V_{\alpha} \nsubseteq P_{\alpha}$.)

While $\mathcal{S}$ is an iteration tree on $\mathcal{J}_{\kappa}^{K}$, we may consider its action on all of $K$. Let $\mathcal{R}$ be the iteration tree on $K$ with the same extenders, drops, and degrees as $\mathcal{S}$. Then $\left(\mathcal{M}_{\beta}^{\mathcal{R}}, E_{\beta}^{\mathcal{T}}\right)$ is weakly countably certified. Hence, by Theorem $2.3, E_{\beta}^{\mathcal{T}}$ is on the $\mathcal{M}_{\beta}^{\mathcal{R}}$-sequence. So $E_{\beta}^{\mathcal{T}}$ is on the $\mathcal{M}_{\beta}^{\mathcal{S}}$-sequence, which is in obvious contradiction with the comparison process.

Now drop the assumption that $\mu^{\omega}=\mu$ and the condition ${ }^{\omega} X_{\alpha} \in X_{\alpha+1}$ above. Instead, we assume that the elementary chain of models is internally approachable; that is, we require that

$$
\left\langle X_{\gamma} \mid \gamma \leq \alpha\right\rangle \in X_{\alpha+1}
$$

for all $\alpha<\kappa$. Define $P_{\alpha}, \pi_{\alpha}$, and $G_{\alpha}$ as in the paragraph above. The difference is that $P_{\alpha}$ is not countably closed. But notice that in the proof of Theorem 2.3, countable closure was only used to see that the $E_{\beta(\alpha)}^{\mathcal{T}}$-extension of $\Phi(\mathcal{R})$ is iterable, so that we could apply [St1, 8.6]. The methods of [MiSch] adapt to show that for a stationary set of $\alpha \in C$, the $E_{\beta(\alpha)}^{\mathcal{T}}$-extension of $\Phi(\mathcal{R})$ is iterable. Below we sketch the main ideas of the proof.

Since $\operatorname{crit}\left(E_{\beta(\alpha)}^{\mathcal{T}}\right)=\alpha$ and $E_{\beta(\alpha)}^{\mathcal{T}}$ measures all sets in $\mathcal{M}_{\alpha}^{\mathcal{R}}, E_{\beta(\alpha)}^{\mathcal{T}}$ is applied to all of $\mathcal{M}_{\alpha}^{\mathcal{R}}$ in forming the $E_{\beta(\alpha)}^{\mathcal{T}}$-extension of $\Phi(\mathcal{R})$.

Let us say that a mouse $\mathcal{Q}$ is $\alpha$-good if replacing the starting model $\mathcal{M}_{\alpha}^{\mathcal{R}}$ of the phalanx $\Phi(\mathcal{R}\lceil(\alpha+1))$ by $\mathcal{Q}$ yields an iterable phalanx. (This corresponds to [MiSch, Definition 2.7].) Let us refer to the phalanx thus obtained by the abbreviated notation $\Phi(\mathcal{R} \mid \alpha) \frown\langle\mathcal{Q}\rangle$. In particular, $\mathcal{M}_{\alpha}^{\mathcal{R}}$ is $\alpha$-good, since $\Phi(\mathcal{R} \mid \alpha) \frown\left\langle\mathcal{M}_{\alpha}^{\mathcal{R}}\right\rangle$ is just the $K$-based phalanx $\Phi(\mathcal{R} \uparrow(\alpha+1))$.

Let us say that an $\alpha$-good mouse $\mathcal{Q}$ lifts badly from $\alpha$ to $\kappa$ if replacing the starting model ult $\left(\mathcal{M}_{\alpha}^{\mathcal{R}}, E_{\beta(\alpha)}^{\mathcal{T}}\right)$ of the $E_{\beta(\alpha)}^{\mathcal{T}}$-extension of $\Phi(\mathcal{R})$ by ult $\left(\mathcal{Q}, G_{\alpha}\right)$ yields a phalanx which is not iterable. (This corresponds to [MiSch, Definition 2.4].) Let us refer to the phalanx thus obtained by the abbreviated notation

$$
\Phi(\mathcal{R})^{\frown}\left\langle\operatorname{ult}\left(\mathcal{Q}, G_{\alpha}\right)\right\rangle \text {. }
$$

Because $G_{\alpha}$ extends $E_{\beta(\alpha)}^{\mathcal{T}}$, if for some $\alpha \in C, \mathcal{M}_{\alpha}^{\mathcal{R}}$ does not lift badly from $\alpha$ to $\kappa$, then the $E_{\beta(\alpha)}^{\mathcal{T}}$-extension of $\Phi(\mathcal{R})$ is iterable. So, we assume that for all $\alpha \in C$, $\mathcal{M}_{\alpha}^{\mathcal{R}}$ lifts badly from $\alpha$ to $\kappa$, and the proof will be complete when we reach a contradiction. 
Suppose that $\mathcal{Q}$ is $\alpha$-good and lifts badly from $\alpha$ to $\kappa$. Then we say that $\mathcal{Q}$ is minimal at $\alpha$ if for every iteration tree $\mathcal{U}$ on $\Phi(\mathcal{R}\lceil\alpha) \frown\langle\mathcal{Q}\rangle$,

- no proper initial segment of $\mathcal{M}_{\infty}^{\mathcal{U}}$ lifts badly from $\alpha$ to $\kappa$, and

- if $\mathcal{M}_{\infty}^{\mathcal{U}}$ lifts badly from $\alpha$ to $\kappa$, then $\mathcal{M}_{\infty}^{\mathcal{U}}$ lies above $\mathcal{Q}$ in the tree order of $\mathcal{U}$ and there is no dropping along the branch from $\mathcal{Q}$ to $\mathcal{M}_{\infty}^{\mathcal{U}}$.

(This definition corresponds to the conclusion of [MiSch, Lemma 3.2].) Starting from

$$
\mathcal{N}_{0}=\mathcal{M}_{\alpha}^{\mathcal{R}}
$$

we can find an $\alpha$-good mouse $\mathcal{Q}_{\alpha}$ which lifts badly from $\alpha$ to $\kappa$ and is minimal at $\alpha$ by the following procedure. If $\mathcal{N}_{i}$ is not minimal at $\alpha$, then let $\mathcal{U}_{i}$ witness that $\mathcal{N}_{i}$ is not minimal at $\alpha$ and let $\mathcal{N}_{i+1}$ be the shortest initial segment of $\mathcal{M}\left(\mathcal{U}_{i}, \infty\right)$ which lifts badly from $\alpha$ to $\kappa$. The iterability of $K$ implies that each $\mathcal{N}_{i}$ is $\alpha$-good. [More precisely, it is the iterability of $K$-based phalanxes which is used here and proved

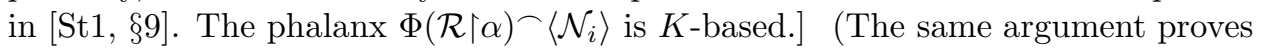
[MiSch, Fact 3.2.1].) Moreover, there is an $i<\omega$ such that $\mathcal{N}_{i+1}$ is not defined, again by the iterability of $K$. [The idea is that we may, in a natural way, consider

$$
\mathcal{R}\left\lceil\alpha \frown \mathcal{U}_{0} \frown \mathcal{U}_{1} \frown \ldots\right.
$$

as an iteration tree on $K$. Moreover, if $\mathcal{U}_{n}$ is defined for every $n<\omega$, then this "concatenation" has no cofinal well-founded branch, either because the only candidate for such a branch has infinitely many drops, or because the underlying tree has no cofinal branches.] (The same argument proves [MiSch, Fact 3.2.2].) Put $\mathcal{Q}_{\alpha}=\mathcal{N}_{i}$ for this $i$. We may assume that $\mathcal{Q}_{\alpha} \in X_{\alpha+1}$.

We can pick functions $f_{k, \alpha}$ for $k<\omega$ and $\alpha \in C$ which represent in ult $\left(\mathcal{M}_{\alpha}^{\mathcal{R}}, E_{\alpha}^{\mathcal{T}}\right)$, a "proof" that $\Phi(\mathcal{R}) \frown\left\langle\operatorname{ult}\left(\mathcal{M}_{\alpha}^{\mathcal{R}}, G_{\alpha}\right)\right\rangle$ is not iterable. [Take a countable elementary submodel

$$
Y \prec V_{\Omega+\omega+\omega}
$$

with everything in it. Let the elements of

$$
Y \cap \operatorname{ult}\left(\mathcal{M}_{\alpha}^{\mathcal{R}}, G_{\alpha}\right)
$$

be represented by the $f_{k, \alpha}$ 's.] Let $\mathcal{M}_{\alpha}$ be the transitive collapse of the hull in $\mathcal{M}_{\alpha}^{\mathcal{R}}$ of

$$
\alpha \cup\left\{f_{k, \alpha} \mid k<\omega\right\} .
$$

Then $\mathcal{M}_{\alpha}$ is still $\alpha$-good and lifts badly from $\alpha$ to $\kappa$.

For $\ell<\omega$, let $\mathcal{M}_{\alpha, \ell}$ be the transitive collapse of the hull in $\mathcal{M}_{\alpha}$ of

$$
\alpha \cup\left\{f_{k, \alpha} \mid k<\ell\right\} .
$$

Each $\mathcal{M}_{\alpha, \ell}$ is coded by a subset of $\alpha$ belonging to $\mathcal{M}_{\alpha}$. [Here we use that $\mathcal{M}_{\alpha}^{\mathcal{R}}$ has height $\Omega$.] So, if $\alpha$ is a limit, then

$$
\mathcal{M}_{\alpha, \ell} \in \operatorname{ran}\left(\pi_{\gamma(\alpha, \ell), \alpha}\right)
$$

for some $\gamma(\alpha, \ell)<\alpha$. Here $\pi_{\gamma, \alpha}$ is the elementary embedding $\pi_{\alpha}^{-1} \circ \pi_{\gamma}$ from $P_{\gamma}$ into $P_{\alpha}$ for all $\gamma<\alpha$. By Fodor's lemma, there is a stationary set $S \subseteq C$ and a fixed $\gamma<\kappa$ such that $\gamma(\alpha, \ell)<\gamma$ for all $\alpha \in S$ and $\ell<\omega$. [Here is where we use that $\aleph_{1}<\kappa$.]

Fix $\alpha \in S$. Say $\pi_{\gamma, \alpha}\left(Z_{\ell}\right)=\mathcal{M}_{\alpha, \ell}$ for $\ell<\omega$. Let $\mathcal{M}^{*}$ be the direct limit of the $Z_{\ell}$ 's under the collapses of the natural maps between the $\mathcal{M}_{\alpha, \ell}$ 's. $\mathcal{M}^{*}$ is called the 
pull-back of $\mathcal{M}_{\alpha}^{\mathcal{R}}$ to $\gamma$. (This corresponds to [MiSch, Lemma 2.1].) Since $\mathcal{M}_{\alpha}$ is $\alpha$ good, $\mathcal{M}^{*}$ is $\gamma$-good. Moreover, the construction of $\mathcal{M}^{*}$ guarantees that since $\mathcal{M}_{\alpha}$ lifts badly from $\alpha$ to $\kappa, \mathcal{M}^{*}$ lifts badly from $\gamma$ to $\kappa$. (This corresponds to [MiSch, Corollary 2.5(b)].) Behind the scenes is the following commutative diagram.

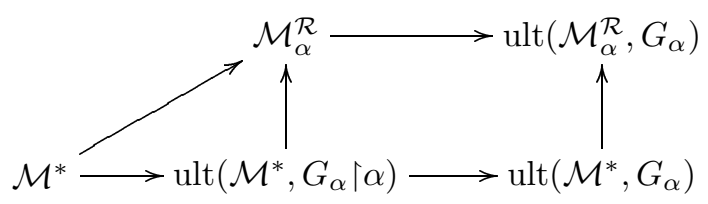

Let us say that a $\gamma$-good mouse $\mathcal{Q}$ lifts badly from $\gamma$ to $\alpha$ if

$$
\Phi\left(\mathcal { R } \lceil \alpha ) \frown \left\langle\operatorname{ult}\left(\mathcal{Q}, G_{\gamma}\lceil\alpha)\right\rangle\right.\right.
$$

is a phalanx which is not iterable.

Since $\operatorname{ult}\left(\mathcal{M}^{*}, G_{\gamma}\lceil\alpha)\right.$ embeds into $\mathcal{M}_{\alpha}^{\mathcal{R}}$ with critical point at least $\alpha$, and $\mathcal{M}_{\alpha}^{\mathcal{R}}$ is $\alpha$-good, $\mathcal{M}^{*}$ does not lift badly from $\gamma$ to $\alpha$. In fact, $\mathcal{M}^{*}$ hereditarily does not lift badly from $\gamma$ to $\alpha$ in the sense that $\mathcal{M}_{\infty}^{\mathcal{V}}$ does not lift badly from $\gamma$ to $\alpha$ whenever

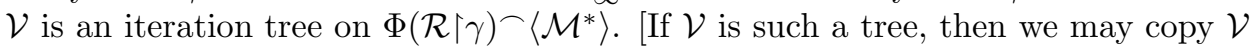
to an iteration tree $\mathcal{W}$ on

$$
\Phi\left(\mathcal { R } \lceil \alpha ) \frown \left\langle\operatorname{ult}\left(\mathcal{M}^{*}, G_{\gamma}\lceil\alpha)\right\rangle,\right.\right.
$$

using the identity maps on models in $\Phi\left(\mathcal{R}\lceil\gamma)\right.$ and the ultrapower map on $\mathcal{M}^{*}$. We may in turn copy $\mathcal{W}$ to an iteration tree $\mathcal{X}$ on $\Phi\left(\mathcal{R}\lceil(\alpha+1))\right.$. Since $\Phi\left(\mathcal{R}\lceil\alpha) \frown\left\langle\mathcal{M}_{\infty}^{\mathcal{X}}\right\rangle\right.$ is $K$-based, it is iterable. Since $\Phi(\mathcal{R} \mid \alpha) \frown\left\langle\mathcal{M}_{\infty}^{\mathcal{W}}\right\rangle$ embeds into $\Phi(\mathcal{R} \mid \alpha) \frown\left\langle\mathcal{M}_{\infty}^{\mathcal{X}}\right\rangle$, it too is iterable.]

On the other hand, $\mathcal{M}_{\gamma}^{\mathcal{R}}$ and $\mathcal{Q}_{\gamma}$ both lift badly from $\gamma$ to $\alpha$. This follows from the fact that they lift badly from $\gamma$ to $\kappa$ and are elements of $X_{\alpha}$. [If $\mathcal{Q}$ is any mouse which lifts badly from $\gamma$ to $\alpha$ and $\mathcal{Q} \in X_{\alpha}$, then $P_{\alpha}$ satisfies that the phalanx

$$
\pi_{\alpha}^{-1}\left(\Phi(\mathcal{R}) \frown\left\langle\operatorname{ult}\left(\mathcal{Q}, G_{\gamma}\right)\right\rangle\right)=\Phi\left(\pi_{\alpha}^{-1}(\mathcal{R})\right) \frown\left\langle\operatorname{ult}\left(\pi_{\alpha}^{-1}(\mathcal{Q}), G_{\gamma}\lceil\alpha)\right\rangle\right.
$$

is not iterable. One can use this to show that $\pi_{\alpha}^{-1}(\mathcal{Q})$ lifts badly from $\gamma$ to $\alpha$. So $\mathcal{Q}$ lifts badly from $\gamma$ to $\alpha$.] (This corresponds to [MiSch, Lemma 2.6].)

Let $(\mathcal{U}, \mathcal{V})$ be the coiteration of $\Phi(\mathcal{R} \mid \gamma)^{\frown}\left\langle Q_{\gamma}\right\rangle$ and $\Phi(\mathcal{R} \mid \gamma)^{\frown}\left\langle\mathcal{M}^{*}\right\rangle$. Since $\mathcal{R}$ is an iteration tree on $K$, using the hull and definability properties and soundness we see that either

(a) $\mathcal{M}_{\infty}^{\mathcal{U}}$ lies above $\mathcal{Q}_{\gamma}$ in the tree order of $\mathcal{U}$, or

(b) $\mathcal{M}_{\infty}^{\mathcal{V}}$ lies above $\mathcal{M}^{*}$ in the tree order of $\mathcal{V}$.

For contradiction, assume that (a) does not hold. Then (b) holds. We claim that $\mathcal{M}_{\infty}^{\mathcal{V}}$ is an initial segment of $\mathcal{M}_{\infty}^{\mathcal{U}}$ and that there is no dropping on the branch from $\mathcal{M}^{*}$ to $\mathcal{M}_{\infty}^{\mathcal{V}}$. The proof is like the proof that $K$ is universal. (Recall that $\mathcal{R}\lceil\gamma$ is an iteration tree on $K$.) From the following commutative diagram, it is clear that $\mathcal{M}_{\infty}^{\mathcal{V}}$ lifts badly from $\gamma$ to $\kappa$, since $\mathcal{M}^{*}$ does.

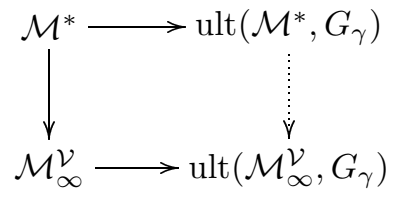

(It is a general fact that if a $\gamma$-good mouse $\mathcal{M}$ which lifts badly from $\gamma$ to $\kappa$ embeds into a mouse $\mathcal{M}^{\prime}$ with critical point at least $\gamma$, then $\mathcal{M}^{\prime}$ also lifts badly from $\gamma$ to 
$\kappa$. This is like [MiSch, Lemma 3.1(a)].) Since $\mathcal{M}_{\infty}^{\mathcal{V}}$ is an initial segment of $\mathcal{M}_{\infty}^{\mathcal{U}}$, $\mathcal{M}_{\infty}^{\mathcal{U}}$ also lifts badly from $\gamma$ to $\kappa$. Since (a) fails, this contradicts the minimality of $\mathcal{Q}_{\gamma}$. Therefore, (a) holds.

We next claim that $\mathcal{M}_{\infty}^{\mathcal{U}}$ is an initial segment of $\mathcal{M}_{\infty}^{\mathcal{V}}$ and that there is no dropping on the branch from $\mathcal{Q}_{\gamma}$ to $\mathcal{M}_{\infty}^{\mathcal{U}}$. If (b) does not hold, then this is essentially the universality of $K$ again. If (b) holds and our claim fails, then there is no dropping on the branch from $\mathcal{M}^{*}$ to $\mathcal{M}_{\infty}^{\mathcal{V}}$. Therefore $\mathcal{M}_{\infty}^{\mathcal{V}}$ is a proper initial segment of $\mathcal{M}_{\infty}^{\mathcal{U}}$ which lifts badly from $\gamma$ to $\kappa$. This contradicts the minimality of $\mathcal{Q}_{\gamma}$.

We have seen that there is an embedding of $\mathcal{Q}_{\gamma}$ into $\mathcal{M}_{\infty}^{\mathcal{U}}$ with critical point at least $\gamma$. From the following commutative diagram, it is clear that $\mathcal{M}_{\infty}^{\mathcal{U}}$ lifts badly from $\gamma$ to $\alpha$, since $\mathcal{Q}_{\gamma}$ does.

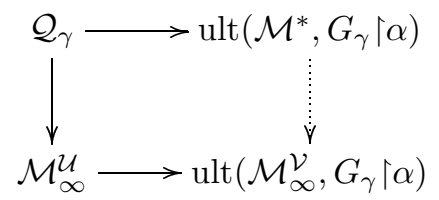

(It is a general fact that if a $\gamma$-good mouse $\mathcal{Q}$ which lifts badly from $\gamma$ to $\alpha$ embeds into a mouse $\mathcal{Q}^{\prime}$ with critical point at least $\gamma$, then $\mathcal{Q}^{\prime}$ also lifts badly from $\gamma$ to $\alpha$. This is like [MiSch, Lemma 3.1(b)].)

Since $\mathcal{M}_{\infty}^{\mathcal{U}}$ is an initial segment of $\mathcal{M}_{\infty}^{\mathcal{V}}, \mathcal{M}_{\infty}^{\mathcal{V}}$ also lifts badly from $\gamma$ to $\alpha$. But $\mathcal{M}_{\infty}^{\mathcal{V}}$ does not lift badly from $\gamma$ to $\alpha$, since, as the reader will recall, $\mathcal{V}$ is an iteration tree on $\Phi(\mathcal{R} \mid \gamma) \frown\left\langle\mathcal{M}^{*}\right\rangle$ and $\mathcal{M}^{*}$ hereditarily does not lift badly from $\gamma$ to $\alpha$. This is a contradiction.

\section{Square PRINCIPLES}

Recall from [Sch1, 5.1] or [Sch2] the following hierarchy of principles intermediate to Jensen's well-known principles $\square_{\kappa}$ and $\square_{\kappa}^{*}$ from [J1]. If $1<\lambda \leq \kappa^{+}$, then $\square_{\kappa}^{<\lambda}$ is the principle asserting the existence of a sequence $\left\langle\mathcal{F}_{\nu}\right| \nu\left\langle\kappa^{+}\right\rangle$such that for every limit ordinal $\nu \in\left(\kappa, \kappa^{+}\right)$,

(1) $1 \leq \operatorname{card}\left(\mathcal{F}_{\nu}\right)<\lambda$, and

(2) for all $C \in \mathcal{F}_{\nu}$,

(a) $C$ is a closed, unbounded subset of $\nu$,

(b) $C$ has order type $\leq \kappa$, and

(c) $C \cap \mu \in \mathcal{F}_{\mu}$ whenever $\mu<\nu$ is a limit point of $C$.

We write $\square_{\kappa}^{\lambda}$ for $\square_{\kappa}^{<\lambda^{+}}$; so $\square_{\kappa} \equiv \square_{\kappa}^{1}$ and $\square_{\kappa}^{*} \equiv \square_{\kappa}^{\kappa}$. Using these forms of weak square, some lower bounds on the large cardinal consistency strength of the Proper Forcing Axiom and other principles were obtained in [Sch1, Sch2, SchSt].

Theorem 3.1 and its proof will be used to prove Theorem 4.1 below.

Theorem 4.1. Suppose that there is no inner model with a Woodin cardinal. Then $\square_{\kappa}^{<\omega}$ holds whenever $\kappa$ is a weakly compact cardinal.

If there is a measurable cardinal $\Omega$, but no inner model with a Woodin cardinal, then $\square_{\kappa}^{<\omega}$ holds for every weakly compact cardinal $\kappa<\Omega$; this is immediate from Theorem 3.1 and [Sch2]. Theorem 4.1 says that the measurable cardinal is not necessary.

Our proof of Theorem 4.1 can be modified to show that if $\kappa$ is a weakly compact cardinal and $\square_{\kappa}^{<\omega}$ fails, then for every $A \subseteq \kappa$ and $n<\omega$ there is an iterable 
inner model which contains $A$ and has $n$ Woodin cardinals. Combining this with [MaSt1] shows that the failure of $\square_{\kappa}^{<\omega}$ at such a $\kappa$ implies PD. Using our proof of Theorem 4.1, Woodin has since shown that if $\kappa$ is weakly compact and $\square \kappa$ fails, then $L(\mathbb{R})$-determinacy holds. Woodin's proof is an induction on the levels of $L(\mathbb{R})$, with Theorem 4.1 as the base case, and he makes the general case look like the base case.

Proof of Theorem 4.1. Suppose that $\kappa$ is a weakly compact cardinal and that there is no inner model with a Woodin cardinal. Assume for contradiction that $\square_{\kappa}^{<\omega}$ fails. Then $A^{\#}$ exists whenever $A$ is a bounded subset of $\kappa$. This follows from Jensen's theorem that $\square_{\kappa}$ holds in $L[A]$ (cf. [J1]) and Kunen's theorem on successors of weakly compact cardinals relativized to $L[A]$ (cf. [Jech, p.384]). Since $\kappa$ is weakly compact, $A^{\#}$ exists for all $A \subseteq \kappa$. Let $\Omega=\kappa^{+}$. Then $\Omega$ is an $A$-indiscernible for every $A \subseteq \kappa$. So, for every $A \subseteq \kappa$, the theory of $K$ up to $\Omega$ of [St1] applies in $L[A]$. Again, we shall write $K^{A}$ for $K^{L[A]}$.

Let $\left\langle A_{\alpha} \mid \alpha<\Omega\right\rangle$ be a sequence of subsets of $\kappa,\left\langle\lambda_{\alpha} \mid \alpha<\Omega\right\rangle$ be a sequence of ordinals, $\left\langle U_{\alpha} \mid \alpha<\Omega\right\rangle$ be a sequence of filters on $\kappa$, and $\left\langle N_{\alpha} \mid \alpha<\Omega\right\rangle$ be a sequence of transitive, power admissible, countably closed sets of cardinality $\kappa$ such that

$$
V_{\kappa} \subset L\left[A_{\alpha}\right]
$$

$A_{\alpha}$ and $U_{\alpha}$ are elements of $L\left[A_{\beta}\right]$,

$$
\lambda_{\alpha}=\left(\kappa^{+}\right)^{K^{A_{\alpha}}}
$$

$U_{\alpha}$ is a nonprincipal, $\kappa$-complete $L\left[A_{\alpha}\right]$-ultrafilter over $\kappa$, and

$$
V_{\kappa} \cup \mathcal{J}_{\lambda_{\alpha}+1}^{K^{A_{\alpha}}} \subset N
$$

whenever $\alpha<\beta<\Omega$. And, also, we assume that $A_{0}$ satisfies the conclusion of Lemma 3.1.1. (As remarked before Lemma 3.1.1, this follows.)

We claim that for unboundedly many $\alpha<\Omega, \lambda_{\alpha} \geq \alpha$. Suppose otherwise, then there is a stationary set $S \subset \Omega$ and a fixed $\lambda<\Omega$ such that $\lambda_{\alpha}=\lambda$ whenever $\alpha \in S$. Fix $\alpha \in S$. Let $A=A_{\alpha}, U=U_{\alpha}$,

$$
j: L[A] \longrightarrow \operatorname{ult}(L[A], U)
$$

be the ultrapower map, $E_{j}$ be the length $j(\kappa)$ extender derived from $j$, and $N=N_{\alpha}$. Note that

$$
\left(\kappa^{+}\right)^{K^{j(A)}}=\lambda
$$

since

$$
\left(\kappa^{+}\right)^{K^{j(A)}} \geq \lambda
$$

and $L[j(A)] \subset L\left[A_{\beta}\right]$ for any $\beta \in S-\alpha$. But now a contradiction follows as in the proof of Theorem 3.1. [If

$$
F=E_{j} \cap\left(\left[j(\kappa]^{<\omega} \times K^{A}\right)\right.
$$

and

$$
G=E_{j} \cap\left(\left[j(\kappa]^{<\omega} \times N\right),\right.
$$

then for unboundedly many $\xi<j(\kappa), L[j(A)]$ satisfies " $(K, F\lceil\xi)$ is countably certified as witnessed by $(N, G)$ ". Then applying Theorem 2.3 gives a Shelah cardinal in $K^{j(A)}$.] 
By Lemma 3.1.1, $K^{A_{\alpha}}$ and $K^{A_{\beta}}$ agree below $\lambda_{\alpha}$ whenever $\alpha<\beta<\Omega$. Let

$$
\vec{E}=\bigcup_{\alpha<\Omega}\left(\dot{E}^{K^{A_{\alpha}}} \uparrow \lambda_{\alpha}\right)
$$

and $\mathcal{M}=\mathcal{J}_{\Omega}^{\vec{E}}$. By the claim of the previous paragraph,

$$
\left(\kappa^{+}\right)^{\mathcal{M}}=\Omega
$$

By [Sch2], $\square_{\kappa}^{<\omega}$ holds in $K^{A_{\alpha}}$ for all $\alpha<\Omega$. Moreover, by [Sch2, Corollary 1.2.1], the witnesses to $\square_{\kappa}^{<\omega}$ can be chosen to be "local definable", so that for each $\alpha<\Omega$, there is a sequence $\mathcal{F}^{\alpha}=\left\langle\mathcal{F}_{\nu}^{\alpha} \mid \nu<\lambda_{\alpha}\right\rangle$ that witnesses $\square_{\kappa}^{<\omega}$ in $K^{A_{\alpha}}$, such that $\mathcal{F}^{\alpha}=\mathcal{F}^{\beta} \mid \lambda_{\alpha}$ whenever $\alpha<\beta<\Omega$. So $\mathcal{F}=\bigcup_{\alpha<\Omega} \mathcal{F}^{\alpha}$ witnesses $\square_{\kappa}^{<\omega}$ in $\mathcal{M}$, and therefore in $V$. This is a contradiction.

If there is a measurable cardinal $\Omega$, but no inner model with a Woodin cardinal, then $\square_{\kappa}^{<\omega}$ holds for every singular cardinal $\kappa<\Omega$. This is immediate from [MiSch] and [Sch2]. The next theorem says that the measurable cardinal is not necessary, at least when $\kappa$ is a singular countably closed cardinal. (Recall that $\kappa$ is countably closed iff $\alpha^{\omega}<\kappa$ whenever $\alpha<\kappa$.)

Theorem 4.2. Suppose that there is no inner model with a Woodin cardinal. Then $\square_{\kappa}^{<\omega}$ holds whenever $\kappa$ is a singular countably closed cardinal.

Jensen proved that if 0 does not exist, then $\square_{\kappa}$ holds whenever $\kappa$ is a singular cardinal. (Cf. [J2] and [J3].) It is not known if Theorem 4.2 holds for all singular cardinals, nor if its conclusion can be strengthened to $\square_{\kappa}$ holding. The proof of Theorem 4.2 can be extended to show that if $\kappa$ is a singular countably closed cardinal and $\square_{\kappa}^{<\omega}$ fails, then for every $A \subseteq \kappa$ and every $n<\omega$, there is an inner model which contains $A$ and has $n$ Woodin cardinals.

Proof of Theorem 4.2. Suppose that $\kappa$ is a singular countably closed cardinal and that there is no inner model with a Woodin cardinal. Assume for contradiction that $\square_{\kappa}^{<\omega}$ fails. Jensen showed that $\square_{\kappa}$ holds in $L[A]$ whenever $A \subseteq \kappa$ (cf. [J1]). Therefore $\left(\kappa^{+}\right)^{L[A]}<\kappa^{+}$whenever $A \subseteq \kappa$.

Lemma 4.2.1. $A^{\#}$ exists whenever $A \subseteq \kappa$.

Sketch. It is clear that Jensen's covering lemma (cf. [DeJ]) can be adapted to show that $A^{\#}$ exists whenever $A$ is a bounded subset of $\kappa$. Suppose that $A$ is an unbounded subset of $\kappa$ and that $\lambda=\left(\kappa^{+}\right)^{L[A]}<\kappa^{+}$. We shall get $A^{\#}$ by piecing together lift-ups of sharps for bounded subsets of $\kappa$.

Let $\pi: N \longrightarrow V_{\theta}$ for some large $\theta$ with $N$ transitive, countably closed, $\operatorname{card}(N)<$ $\kappa$, and $\pi$ cofinal in $\lambda$. Say $\pi(\bar{A})=A, \pi(\bar{\kappa})=\kappa$, and $\pi(\bar{\lambda})=\lambda$. Let $E$ be the length $\kappa$ extender derived from $\pi$. If $\bar{\lambda}$ is not a cardinal of $L[\bar{A}]$, then as usual we let $\mathcal{M}$ be the first level of $L[\bar{A}]$ which sees this; note that

$$
\mathcal{M}=\mathcal{H}_{n+1}^{\mathcal{M}}\left(\bar{\kappa} \cup p_{n+1}^{\mathcal{M}}\right)
$$

for an appropriate $n<\omega$ (fine structure works above $\bar{\kappa}$ ), set $\mathcal{M}^{*}=\operatorname{ult}_{n}(\mathcal{M}, E)$, and get that $\mathcal{M}^{*}$ is a level of $L[A]$ collapsing $\lambda$.

So $\bar{\lambda}$ is a cardinal of $L[\bar{A}]$, and thus $E$ measures all subsets of $\bar{\kappa}$ in $L[\bar{A}]$. Let $\pi^{*}: L[\bar{A}] \longrightarrow \operatorname{ult}(L[\bar{A}], E)=L[A]$ be the lift-up of $\pi$. Let $D$ be the class of strong limit cards of cofinality $\kappa^{+}$. Note the tuples from $D$ are $\bar{A}$-indiscernible with respect to parameters $<\bar{\kappa}$. Also, $\pi^{*}(\alpha)=\alpha$ for $\alpha \in D$. By the elementarity of $\pi^{*}$, the 
tuples from $D$ are $A$-indiscernible with respect to parameters from $\kappa$. Since $D$ is stationary, we are done.

Let $\vec{\kappa}$ be an increasing sequence of cardinals cofinal in $\kappa, \Omega=\kappa^{+}$, and $A$ be as in Lemma 3.1.1. Choose $\left\langle A_{\alpha} \mid \alpha<\Omega\right\rangle$ and $\left\langle\lambda_{\alpha} \mid \alpha<\Omega\right\rangle$ so that

$$
\vec{\kappa}, A \in L\left[A_{0}\right]
$$

and whenever $\alpha<\beta<\Omega$,

$$
\begin{gathered}
A_{\alpha} \in L\left[A_{\beta}\right], \\
\left(\kappa^{+}\right)^{L\left[A_{\alpha}\right]}<\left(\kappa^{+}\right)^{L\left[A_{\beta}\right]},
\end{gathered}
$$

and

$$
\lambda_{\alpha}=\left(\kappa^{+}\right)^{K^{A_{\alpha}}} .
$$

Then, for every $\alpha<\Omega, \kappa$ is singular in $L\left[A_{\alpha}\right]$, so, by the weak covering theorem of [MiSch] applied in $L\left[A_{\alpha}\right]$,

$$
\left(\kappa^{+}\right)^{L\left[A_{\alpha}\right]}=\lambda_{\alpha} .
$$

The rest of the proof is just like that of Theorem 4.1.

\section{AN ERdÖs PROPERTY}

It has been observed that the existence of sharps for all sets (rather than a measurable cardinal $\Omega$ ) suffices to develop the theory in [St1], and consequently, to prove a version of the results in this paper. (Cf. [St1, p.58].) Theorem 5.1 below, an improvement to Theorem 3.1, illustrates how an Erdös partition property on $\Omega$ consistent with $V=L$ suffices in many core model applications.

Let $\widetilde{K}$ be the premouse determined by the inductive definition of the core model in [St1, $\S 6]$. By [St1], if $\Omega$ is a measurable cardinal, then $K=\widetilde{K}$ up to $\Omega$, but it is not known if one can show in ZFC that $\widetilde{K}$ is a proper class.

Theorem 5.1. Suppose that there is no inner model with a Woodin cardinal and that $\kappa<\Omega$ are cardinals satisfying the partition relation $\Omega \longrightarrow(\omega)_{\kappa}^{<\omega}$. Suppose that $\kappa$ is a weakly compact cardinal. Then $\widetilde{K}$ is a premouse of height $>\kappa^{+}$and $\left(\kappa^{+}\right)^{\widetilde{K}}=\kappa^{+}$.

Of course, Theorem 3.1 is just a special case of Theorem 5.1. The same method of proof can be used to show that the hypothesis

$$
\Omega \longrightarrow(\omega)_{\kappa}^{<\omega}
$$

for all $\kappa<\Omega$, is more than enough for all applications in [MiSch], [MiSchSt], [Sch1], [Sch2], [St1], and [St3]. In fact, the proof uses only a model theoretic consequence of the partition relation.

Proof of Theorem 5.1. Suppose that $\kappa<\Omega$ are cardinals such that $\kappa$ is weakly compact and

$$
\Omega \longrightarrow(\omega)_{\kappa}^{<\omega} .
$$

Assume that there is no inner model with a Woodin cardinal.

We shall adapt an argument of Reinhardt and Silver; see [Jech, pp.394-395]. Let $\mathfrak{A}$ be the structure $\left\langle V_{\Omega}, \in, \kappa\right\rangle$ expanded to include predicates for Skolem functions. 
Let $I$ be an infinite set of indiscernibles for $\mathfrak{A}$ such that $I \subset(\kappa, \Omega)$. The existence of $I$ follows from our assumption that $\Omega \longrightarrow(\omega)_{\kappa}^{<\omega}$ in the standard way. Let

$$
\pi: \mathfrak{B} \longrightarrow \mathfrak{A}
$$

be the inverse transitive collapse of the closure of $I$ under the Skolem functions for $\mathfrak{A}$. Fix some $\alpha \in I$ and put $\bar{\alpha}=\pi^{-1}(\alpha)$ and $\bar{\kappa}=\pi^{-1}(\kappa)$. Let $j: \mathfrak{B} \longrightarrow \mathfrak{B}$ be an elementary embedding obtained by "shifting" $\bar{\alpha}$, so that $\operatorname{crit}(j) \leq \bar{\alpha}$. With a judicious choice of $I$, we can arrange that $\operatorname{crit}(j)>\bar{\kappa}$.

Now, using $j$ just as the embedding from a normal measure was used in [St1], one can show that the inductive definition of the core model, as carried out in $\mathfrak{B}$, succeeds up to $\operatorname{crit}(j)$. (The details are just like those described in [St1, p.58]. Recall that [St1, $\S 1-\S 6, \S 9]$ develops the theory of $K^{c}$ and $K$ in $V$ up to a measurable cardinal while working over a structure $\langle V, \epsilon, U\rangle$ where $U$ is a normal measure on the measurable cardinal. Let $U_{j}$ be the $\mathfrak{B}$-ultrafilter on $\operatorname{crit}(j)$ given by $X \in U_{j}$ iff $\operatorname{crit}(j) \in j(X)$. Then $U_{j}$ is weakly amenable to $\mathfrak{B}$. Now construct $K^{c}$ and $K$ up to $\operatorname{crit}(j)$ over the structure $\left\langle|\mathfrak{B}|, \epsilon, U_{j}\right\rangle$. The proofs in [St1, $\left.\S \S 1-6, \S 9\right]$ go through with only obvious changes to show that the construction succeeds.)

Since $\pi$ is an elementary embedding, $\widetilde{K}$ has height $\geq j(\operatorname{crit}(j))>\kappa^{+}$. Arguing as in Theorem 3.1, we see that

$$
\mathfrak{B} \models\left(\bar{\kappa}^{+}\right)^{K}=\bar{\kappa}^{+} .
$$

By elementarity again, we conclude that $\left(\kappa^{+}\right)^{\widetilde{K}}=\kappa^{+}$.

\section{REFERENCES}

[DeJ] K.I. Devlin and R.B. Jensen, Marginalia to a theorem of Silver, in Logic Conference, Kiel 1974, Lecture Notes in Mathematics, 499, Springer-Verlag, 1975, 115-142. MR 58:235

[Do1] A.J. Dodd, Strong Cardinals, circulated notes, 1981.

[Do2] A.J. Dodd, The core model, London Mathematical Society Lecture Note Series, 61, Cambridge University Press, Cambridge-New York, 1982. MR 84a:03062

[DoJ] A.J. Dodd and R.B. Jensen, The core model, Ann. Math. Logic 20 (1981), no. 1, 43-75. MR 82i:03063

[Jech] T. Jech, Set theory, Academic Press, New York-London, 1978. MR 80a:03062

[J1] R.B. Jensen, The fine structure of the constructible hierarchy, Ann. Math. Logic 4 (1972), no. 3, 229-308. MR 46:8834

[J2] R.B. Jensen, Non overlapping extenders, circulated notes.

[J3] R.B. Jensen, Some remarks on $\square$ below 0 ${ }^{\top}$, circulated notes.

[MaSt1] D.A. Martin and J.R. Steel, A proof of projective determinacy, J. Amer. Math. Soc. 2 (1989) 71-125. MR 89m:03042

[MaSt2] D.A. Martin and J.R. Steel, Iteration trees, J. Amer. Math. Soc. 7 (1994) 1-73. MR 94f:03062

[Mi] W.J. Mitchell, The core model for sequences of measures I, Math. Proc. Cambridge Philos. Soc. 95 (1984), no. 2, 229-260. MR 85i:03163

[MiSch] W.J. Mitchell and E. Schimmerling, Weak covering without countable closure, Math. Res. Lett. 2 (1995), no. 5, 595-609. MR 96k:03123

[MiSchSt] W.J. Mitchell, E. Schimmerling, and J.R. Steel, The covering lemma up to a Woodin cardinal, Ann. Pure Appl. Logic 84 (1997), no. 2, 219-255. MR 98b:03067

[MiSt] W.J. Mitchell and J.R. Steel, Fine structure and iteration trees, Lecture Notes in Logic 3, Springer-Verlag, Berlin, 1994. MR 95m:03099

[Sch1] E. Schimmerling, Combinatorial principles in the core model for one Woodin cardinal, Ann. Pure Appl. Logic 74 (1995), no. 2, 153-201. MR 96f:03041

[Sch2] E. Schimmerling, A finite family weak square principle, to appear in J. Symbolic Logic. 
[SchSt] E. Schimmerling and J.R. Steel, Fine structure for tame inner models, J. Symbolic Logic 61 (1996), no. 2, 621-639. MR 97c:03123

[SchW] E. Schimmerling and W.H. Woodin, The Jensen covering property, to appear in J. Symbolic Logic.

[St1] J.R. Steel, The core model iterability problem, Lecture Notes in Logic 8, SpringerVerlag, Berlin, 1996. CMP 98:04

[St2] J.R. Steel, Inner models with many Woodin cardinals, Ann. Pure Appl. Logic 65 (1993), no. 2, 185-209. MR 95c:03132

[St3] J.R. Steel, Core models with more Woodin cardinals, preprint.

Department of Mathematics, University of California, Irvine, Irvine, California 92697-3875

Current address: Mathematical Sciences Department, Carnegie Mellon University, Pittsburgh, Pennsylvania 15213

E-mail address: eschimme@andrew.cmu.edu

Department of Mathematics, University of California, Berkeley, Berkeley, CaliFORNIA 94720

E-mail address: steel@math.berkeley.edu 